\title{
¿Surface Gravity Wave Effects on Submesoscale Currents in the Open Ocean
}

\author{
Delphine Hypolite, ${ }^{\mathrm{a}}$ Leonel Romero, ${ }^{\mathrm{b}}$ James C. McWilliams, ${ }^{\mathrm{a}}$ And Daniel P. DauhajRe ${ }^{\mathrm{a}}$

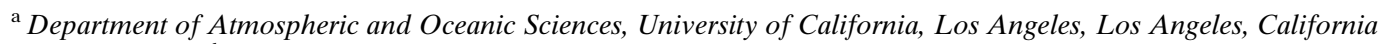 \\ ${ }^{\mathrm{b}}$ Department of Marine Sciences, University of Connecticut, Groton, Connecticut
}

(Manuscript received 14 December 2020, in final form 25 August 2021)

\begin{abstract}
A set of realistic coastal simulations in California allows for the exploration of surface gravity wave effects on currents (WEC) in an active submesoscale current regime. We use a new method that takes into account the full surface gravity wave spectrum and produces larger Stokes drift than the monochromatic peak-wave approximation. We investigate two high-wave events lasting several days-one from a remotely generated swell and another associated with local windgenerated waves - and perform a systematic comparison between solutions with and without WEC at two submesoscaleresolving horizontal grid resolutions $(d x=270$ and $100 \mathrm{~m})$. WEC results in the enhancement of open-ocean surface density and velocity gradients when the averaged significant wave height $H_{s}$ is relatively large $(>4.2 \mathrm{~m})$. For smaller waves, WEC is a minor effect overall. For the remote swell (strong waves and weak winds), WEC maintains submesoscale structures and accentuates the cyclonic vorticity and horizontal convergence skewness of submesoscale fronts and filaments. The vertical enstrophy $\zeta^{2}$ budget in cyclonic regions $(\zeta / f>2)$ reveals enhanced vertical shear and enstrophy production via vortex tilting and stretching. Wind-forced waves also enhance surface gradients, up to the point where they generate a smallsubmesoscale roll-cell pattern with high vorticity and divergence that extends vertically through the entire mixed layer. The emergence of these roll cells results in a buoyancy gradient sink near the surface that causes a modest reduction in the typically large submesoscale density gradients.
\end{abstract}

KEYWORDS: Waves, oceanic; Small scale processes; Frontogenesis/frontolysis

\section{Introduction}

Surface gravity waves are known to have a strong dynamical coupling with oceanic currents in at least two situations: in the wind-driven surface boundary layer where they engender Langmuir turbulence (LT) (Langmuir 1938; McWilliams et al. 1997; Thorpe 2004; Sullivan and McWilliams 2010; Belcher et al. 2012), and in the surf zone where breaking induces littoral (rip) currents (Longuet-Higgins and Stewart 1964; Feddersen and Trowbridge 2005; MacMahan et al. 2006; Marchesiello et al. 2015; Uchiyama et al. 2017). In both regimes a waveaverage theory of the effects of waves on currents (WEC) (Craik and Leibovich 1976; McWilliams et al. 2004; Suzuki and Fox-Kemper 2016) has been shown to yield useful and realistic simulations. In this paper we explore WEC for upper-ocean currents by an extension of this method.

The horizontal length scales $L$ involved in LT are small $[\leq \mathscr{O}(100) \mathrm{m}]$ and partly overlap with the scales of submesoscale currents (McWilliams 2016). The submesoscale regime manifests when the local Rossby number Ro $=V /(f L)(V$ is a typical horizontal velocity scale and $f$ the Coriolis frequency) is $\mathscr{O}(1)$, with the emergence of short-lived (hours to days), intermediatesized $\odot(0.1-10) \mathrm{km}$ flow structures of density fronts and filaments, and coherent vortices. Such fronts and filaments are characterized by strong surface cyclonic vorticity, horizontal

\footnotetext{
๑ Denotes content that is immediately available upon publication as open access.
}

Corresponding author: Delphine Hypolite, dhypolite@atmos. ucla.edu convergence, vertical velocity, and horizontal density gradients (Mahadevan and Tandon 2006; Nagai et al. 2006; Capet et al. 2008a,c; Gula et al. 2014; Bracco et al. 2016; Wang et al. 2021). These structures play a significant role in the transport of materials nearshore (Romero et al. 2013; Dauhajre et al. 2019) and in the upper open-ocean (Goodman 2012; Lévy et al. 2012; Zhong and Bracco 2013; Mahadevan 2016).

In this context, numerical large-eddy simulations (LES) that explicitly calculate the boundary layer turbulence have proved useful for resolving relevant dynamical scales involved in LT and even in some pioneering studies for their interaction with submesoscale currents (Sundermeyer et al. 2014; Hamlington et al. 2014; Sullivan and McWilliams 2019). However, such studies rely on simplified conditions such as flat bathymetry and horizontally uniform wind stress and wave Stokes drift, thus encompassing only a limited view of submesoscale currents and their spatial inhomogeneity and temporal nonstationarity. Also, in previous idealized studies of WEC, the simplifying assumption of wind-wave equilibrium is often made, even though it is not pervasive in nature (Hanley et al. 2010), and often the wave forcing is simplified to a spectrumpeak monochromatic wave field (Sundermeyer et al. 2014).

To overcome these limitations we instead use a high-resolution circulation model with a parameterization for the effects of boundary layer turbulence. Our code is the Regional Oceanic Modeling System (ROMS; Shchepetkin and McWilliams 2005), including WEC (Uchiyama et al. 2010). A key aspect of this work is that we use reanalysis wind and wave forcings that are broad band and not assumed to be in equilibrium. A companion study of current effects on waves (CEW) is Romero et al. (2020), although we do not include a full CEW-WEC coupling here. Previous investigations of realistic, coupled 


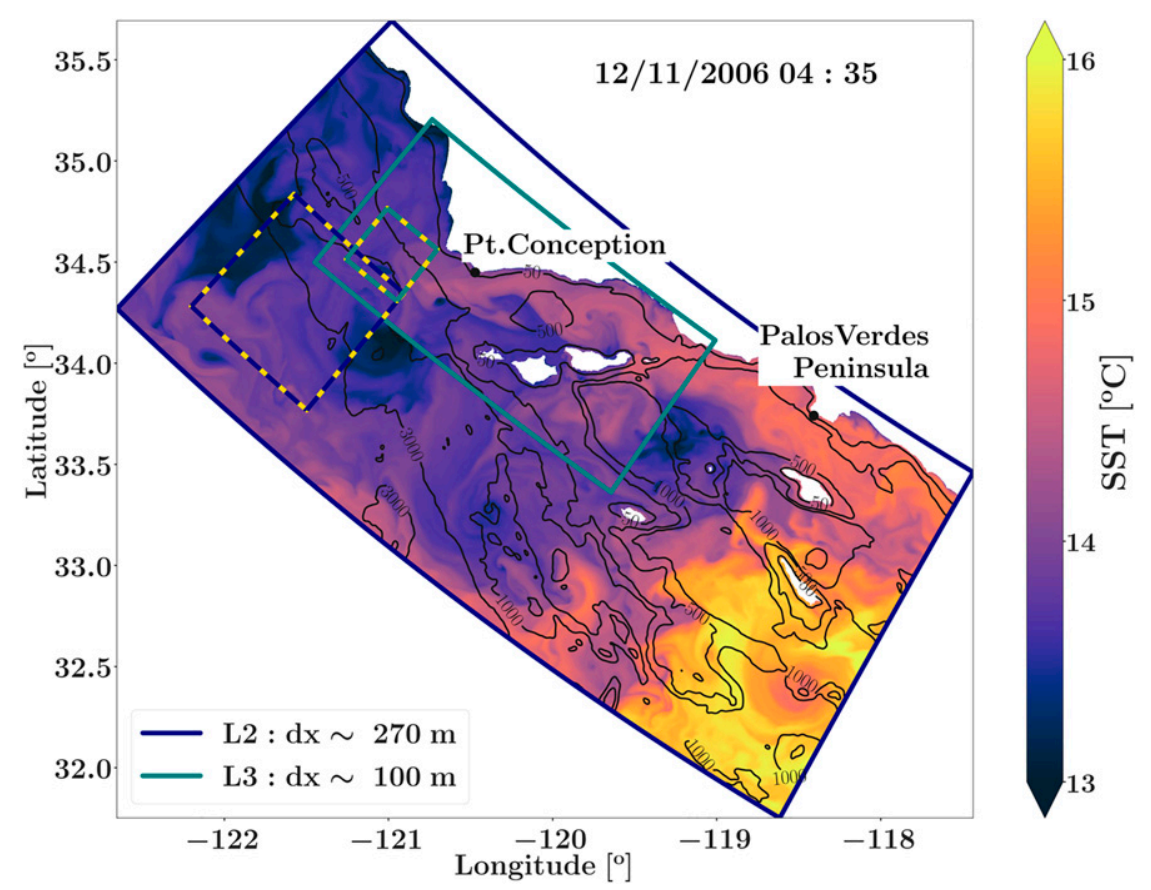

FIG. 1. Regional Oceanic Modeling System (ROMS) domains in MidCal at two horizontal resolutions: the L2 domain delimited in blue with an average horizontal spacing $d x=270 \mathrm{~m}$ and the L3 domain in teal with $d x=100 \mathrm{~m}$. Simulated sea surface temperature (SST) is plotted as a snapshot at 0435 local time11 Dec 2006. The dashed boxes show sampled areas used for the statistical analysis of the open ocean. We plot in black the 50-, 500-, 1000-, and 3000-m isobaths.

wave-current interaction have focused on estuary (Olabarrieta et al. 2011) and nearshore (Kumar et al. 2012) regions, although neither have explored wave effects on submesoscale flows.

The present paper is primarily an exploration of the WEC possibilities for submesoscale currents. The theoretical framework for interpreting this interaction is limited. It includes the ideas of an advective vortex force, an augmented Coriolis force, and material concentration advection-all related to the wave Stokes drift current. Often this leads to both an Eulerian current partly in opposition to the Stokes drift and to modified frontogenetic secondary circulations around fronts and filaments (McWilliams 2018).

In section 2 we describe the oceanic model configuration used to produce a family of middle California (MidCal) solutions at two submesoscale resolutions: $d x=270$ and $100 \mathrm{~m}$. We explain the WEC implementation in ROMS, which includes a representation of the wave Stokes drift velocity obtained from realistic wave solutions (Romero et al. 2021). In section 3 we provide a statistical comparison of submesoscale fields from solutions with and without WEC. In section 4 we give a dynamical interpretation of WEC statistical properties by investigating the dynamical balances for vertical and horizontal enstrophy, squared horizontal buoyancy gradient, and horizontal divergence, including WEC for the first time. This analysis allows the identification of the mechanism by which waves, when big enough, reinforce submesoscale activity (frontogenesis) near the surface. Similarly, we show how windforced waves can produce roll-cell currents that disrupt classic submesoscale features. In section 5 we illustrate WEC effects in an individual submesoscale filament by decomposing the momentum balance in the along/across-filament frame to show the relative importance of the vortex force. We discuss the resemblance of the roll cells similar to Langmuir cells in LT that emerge when there are large wind-forced waves. Finally, we provide kinetic energy conversion estimates in different wave regimes. Results are summarized and discussed in section 6 .

\section{Simulation setup}

\section{a. ROMS configuration}

We simulate the 3D oceanic circulation in the MidCal coastal sector using ROMS. It solves the hydrostatic primitive equations, where the vertical mixing relies on a $K$-profile parameterization (Large et al. 1994). The parent solutions (referred to as L0 and L1, with $d x=4$ and $1 \mathrm{~km}$ horizontal resolution, respectively), encompassing the full U.S. West Coast, are downscaled successively with a one-way nesting technique (Mason et al. 2010) to produce two finer, submesoscale-resolving (Capet et al. 2008a) horizontal grid resolutions: first a $d x=$ 270-m horizontal resolution (called L2), and then a $d x=100 \mathrm{~m}$ horizontal resolution (L3). We show the two corresponding domains in Fig. 1. The grids are centered on the Channel Islands and capture the physical variability of the areas surrounding Point Conception, the Channel Islands, and the Santa 

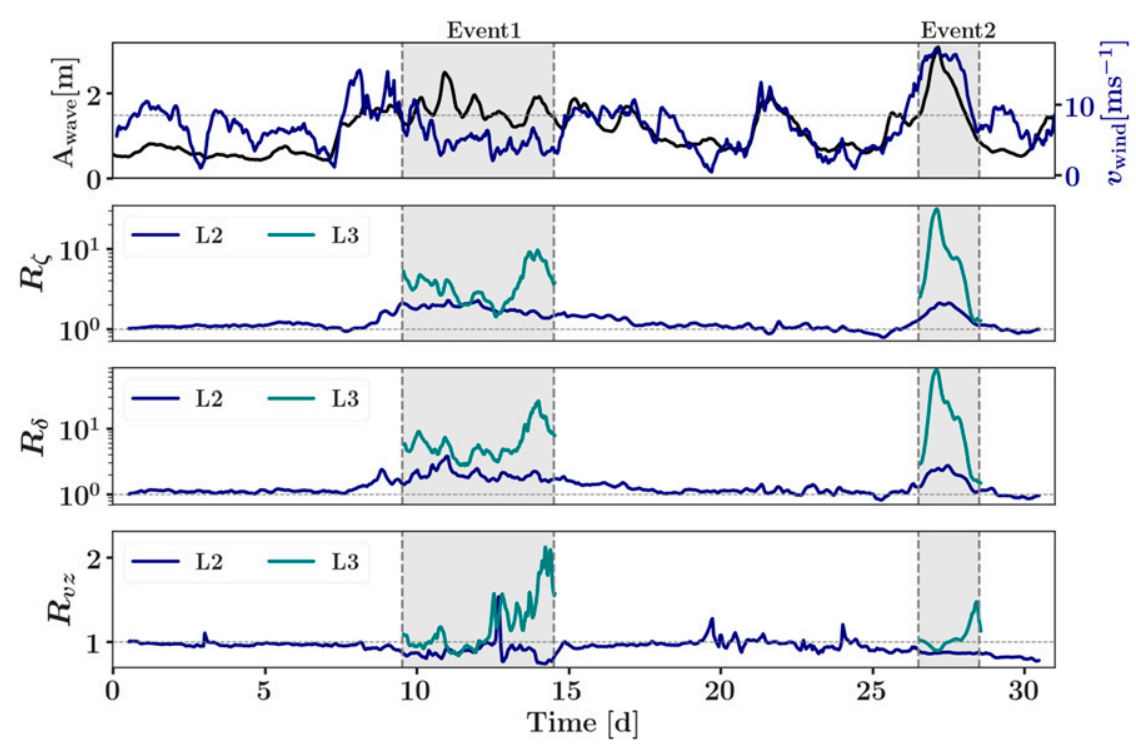

FIG. 2. Time series (days since 0000 local time 1 Dec 2006) of (first row) wind speed $v_{\text {wind }}$ and wave amplitude $A_{\text {wave }}$ (blue and black, respectively), squared normalized (second row) vorticity and (third row) divergence at $1 \mathrm{~m}$ below the surface, and (fourth row) squared vertical shear vertically integrated over the first $10 \mathrm{~m}$. The latter three panels are shown as the RMS ratios $R$ of solutions with and without WEC in the L2 (blue) and L3 (teal) solutions during December 2006. L3 solutions are only displayed during high-wave events, i.e., when $A_{\text {wave }} \gtrsim 1.5 \mathrm{~m}$. Fields are horizontally averaged in the sampled areas shown in Fig. 1 .

Barbara Channel. The present study focuses solely on the open-ocean dynamics (areas delimited by dashed lines in Fig. 1). This ROMS configuration is from the same family of solutions as analyzed in Dauhajre et al. (2019), containing the same realistic bathymetry, tidal variability (Buijsman et al. 2012), and atmospheric forcing. Tides are included in the parent L1 solution and pass through the boundary conditions to both $\mathrm{L} 2$ and L3. The nests are forced hourly by realistic atmospheric fields and fluxes (precipitation, solar radiation, wind, and air temperature and humidity) interpolated from a parent $d x=6 \mathrm{~km}$ horizontal resolution Weather Research and Forecast (WRF) Model (Michalakes et al. 1998) solution, derived from the NCEP NARR database. Atmospheric fluxes are parameterized according to Large (2006) and account for surface ocean currents through a wind-current coupling parameterization that produces a more realistic eddy kinetic energy level (Renault et al. 2016; Renault et al. 2021).

Simulations are performed first without WEC in L2 and L3. When the WEC capability is enabled in ROMS (section 2b), WEC solutions are produced with the same boundary conditions. The L2 lower-resolution analysis presented here is based on 1-h output frequency of instantaneous fields and simulates the month of December 2006. We choose this wintertime period due to a combination of rough weather, associated with large-wave-amplitude events, with a less stratified upper ocean (compared to summer) that energizes more active submesoscale dynamics (Callies et al. 2015). The L3 higher-resolution analysis utilizes a higher output frequency of $30 \mathrm{~min}$ of instantaneous fields and targets two specific large-wave-amplitude events in December 2006 (event 1: 9-14 December 2006, event 2: 27-29 December 2006; see Fig. 2 and section 3a). For these L3 simulations we start the integrations 2 days (observed adjustment time of the surface layer dynamical balance) prior to each high-wave event to allow the solution to adjust to the grid-size change and only analyze the solution during the events.

\section{b. WEC implementation in ROMS}

Originally WEC was implemented in ROMS by Uchiyama et al. (2010), within the asymptotic theoretical assumption of a small wave slope framework established by McWilliams et al. (2004). It uses the vortex force formalism, for conservative wave-averaged dynamical effects of waves acting on currents, in combination with accelerations due to wave breaking and wave-induced mixing effects. It results in the addition of the conservative Stokes drift vortex force and material advection, the Stokes-Coriolis force, the wave-induced pressure corrections (such as the Bernouilli head or in the surface boundary conditions), and the quasi-static sea level setup. Other WEC contributions are nonconservative, such as waveinduced mixing of momentum and tracers, bottom drag, and breaking, some of which are negligible in the open ocean. However, these simulations were made with the conventional $K$-profile parameterization without its LT augmentation ( $\mathrm{Li}$ et al. 2019); this modification is left for future work. These WEC contributions are computed in accordance with a given wave field. The wave field is usually determined by a spectral numerical wave model such as WAVEWATCH 3 (WW3; Tolman 2009), which provides wave parameters that are passed 
to ROMS to compute the WEC contributions, e.g., Kumar et al. (2012).

Previous uses of the ROMS-WEC model relied on the spectrum-peak approximation which produces a generally small Stokes drift near the surface in the open ocean (compared to full-spectrum WW3 estimates) (Romero et al. 2021). To remedy this underestimation, we utilize here a more realistic approach for calculating Stokes drift that respects the broad wavenumber and frequency spectra typical of observed surface gravity waves, thus departing from the original WEC implementation of Uchiyama et al. (2010). The setup of the WW3 model we use is the same as in Romero et al. (2020), calculated on the L2 and L3 grids. This method, described by Romero et al. (2021), uses the surface Stokes drift and Stokes transport resulting from the full wave spectrum integration in WW3 to approximate the vertical profile of Stokes drift, which is similar to the work by Breivik et al. (2014) but not limited to deep-water waves. Therefore, it is better suited for coastal applications and can better handle conditions with mixed wind sea and swell. It allows a variety of wave events to produce different Stokes drift intensities and vertical structures. Highfrequency wave spectrum components (generally excited by wind) contribute primarily near the surface, and lower-frequency contributions, such as from remote swells, affect the Stokes drift profile deeper (Tamura et al. 2012). For comparable surface Stokes drift velocities, the vertical extent of the Stokes drift is therefore usually larger for swell events from large distant storms than it is for wind-forced waves. In the MidCal region we expect the westerlies to generate Stokes drift mainly eastward. In addition, offshore storms generate oceanic waves, and during the winter season we expect them mainly from the interior North Pacific, thus propagating eastward as swell waves. These remote swell events are particularly susceptible to misalignment with the local wind direction.

\section{WEC phenomena}

\section{a. Wave amplitude dependency}

Figure 2 shows a time series of the horizontally averaged wind and wave forcings (see the first row) that are used in L2 and L3 during the month of December 2006. The spatial averaging is performed within the respective dashed boxes in Fig. 1 that exclusively sample the open-ocean dynamics (results are similar when performing the averages in only the L3 subdomain). The L3 atmospheric forcing fields are interpolated from L2 and therefore have equivalent time series. We identify two periods of time when the averaged wave amplitude $A_{\text {wave }}$ gets larger than about $1.5 \mathrm{~m}$ (horizontal gray dashed line): one period in between 9 and 14 December 2006 ( 5 days starting at 0400 local time), and another starting on 27 December 2006 that lasts $48 \mathrm{~h}$ ( 2 days starting at 0400 local time); we refer to these high-wave periods as events 1 and 2, respectively, and they are gray shaded in Fig. 2. They are associated with a surface Stokes drift amplitude of $0.1 \mathrm{~m} \mathrm{~s}^{-1}$ or higher. The two events differ in their wind intensity and corresponding wave spectrum. During event 1 the local wind is weak with a variable direction, and the waves are mostly remotely generated swell.
Event 2 is a combination of strong westerly wind and largeamplitude waves, which arise from local wind generation. In both events the Stokes drift is mainly eastward. Because of the strong wind we expect event 2 to display dynamics that departs from the familiar submesoscale framework with or without WEC, as discussed in Sun et al. (2020), where they demonstrate that wind bursts can erode submesoscale vorticity and divergence.

We compare fields indicative of submesoscale flows for WEC and NO WEC solutions by computing the ratio of the spatially averaged variances of vertical vorticity, $\zeta=v_{x}-u_{y}$, and horizontal divergence, $\delta=u_{x}+v_{y}, 1 \mathrm{~m}$ below the surface, and of vertical shear variance, $\left|\mathbf{v}_{z}^{h}\right|^{2}=\left(u_{z}^{2}+v_{z}^{2}\right)$, integrated over the first $10 \mathrm{~m}$. The $3 \mathrm{D}$ Cartesian velocity components are $\mathbf{v}=$ $(u, v, w)$, and a vector superscript $h$ denotes the horizontal components. These ratios are designated by $R_{\zeta}, R_{\delta}$, and $R_{v z}$. Note that the variances of $\zeta$ and $\mathbf{v}_{z}$ are, respectively, the vertical component and the horizontal component of the enstrophy (the latter in a hydrostatic approximation).

When the wave amplitude $\left(A_{\text {wave }}=H_{s} / 2 \sqrt{2}\right)$ is small with $A_{\text {wave }}<1.5 \mathrm{~m}$, the ratio of the three fields is close to unity, meaning that, on average, the solutions with and without wave forcing have velocity gradients of comparable amplitude (e.g., days $0-10$ in Fig. 2). For small waves the solutions with WEC are similar to the solutions without WEC. During the highwave events (shaded areas in Fig. 2), ratios of surface velocity gradients become larger than one, implying a departure from "typically" discussed submesoscale dynamics (i.e., without WEC). This applies to related quantities such as vertical velocity and buoyancy gradients, but these are not shown here. WEC enhances velocity and density gradients near the surface where Stokes drift is important. This effect is accentuated at the L3 level. The ratios reach 30 times higher enstrophy and 70 times higher squared divergence for the L3 WEC solutions. We also observe that the squared vertical shear is enhanced with WEC during both events at L3. In particular, peaks of vertical shear are concomitant with peaks of divergence and enstrophy during event 1.

\section{b. WEC statistical impacts}

We illustrate the WEC enhancement of near-surface velocity gradients with snapshots of surface normalized vorticity in Fig. 3. We compare the solution with wave forcing to the solution without waves in the L2 domain at 1600 local time 28 December 2006, during event 2. The solution without WEC forcing displays familiar properties of submesoscale dynamics with a mixture of fronts, vortices, and filaments with large, positive vorticity $(\zeta / f \gg 1)$. The NO WEC solution favors cyclonic structures with a skewness of 2.0 for the two high-wave events combined. The solution that includes WEC has stronger vorticity, more visible on cyclonic structures. The skewness of the WEC solution is 3.1 during the high-wave events. The submesoscale dynamics is also favoring convergent structures, and the skewness in normalized divergence is -1.7 without WEC and -2.0 with WEC.

Figure 4 further illustrates more highly resolved WEC effects on submesoscale currents in the L3 domain. WEC and NO WEC solutions tend to decorrelate with time. However, by initializing each L3 simulation close to the beginning of the 


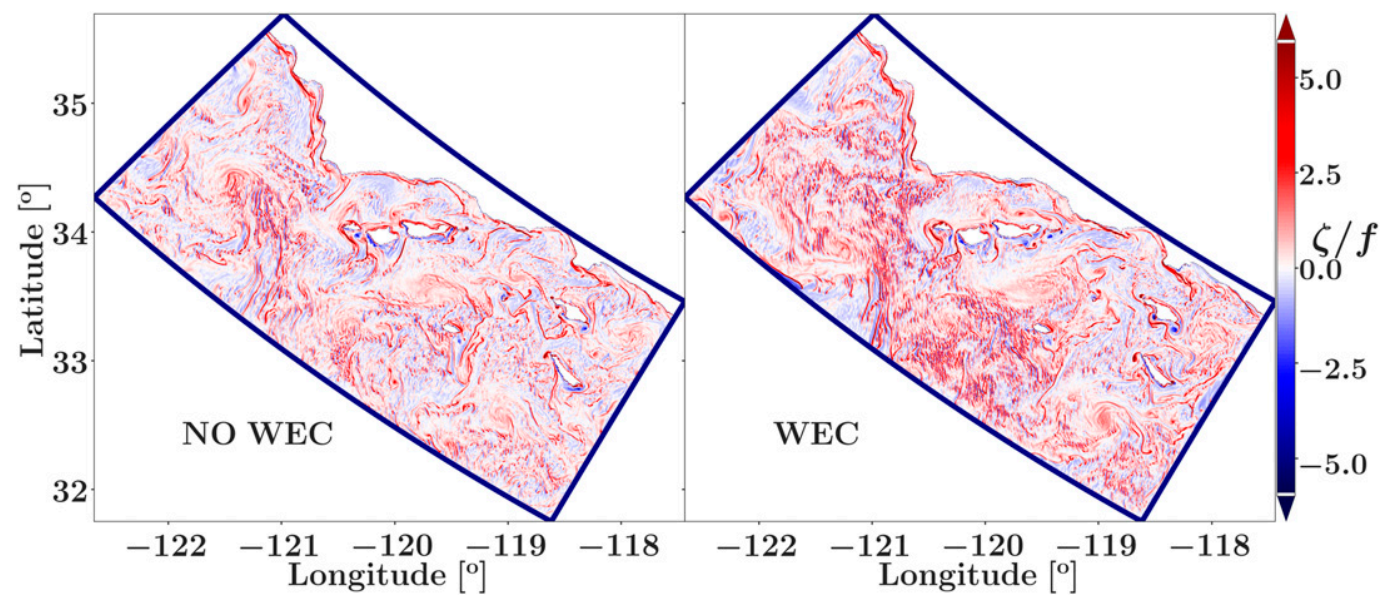

FIG. 3. Snapshots of surface normalized vorticity in the full L2 domain at 1600 local time 28 Dec 2006 (event 2). (left) The solution without wave forcing and (right) the solution with WEC. Regions of cyclonic vorticity are in red (anticyclonic regions are in blue). Note that the color bar is saturated.

high-wave events, a limited period of correlation allows direct comparisons of individual structures between WEC and NO WEC solutions, particularly when the wind is weak. For instance, it is possible to follow, from the same initial conditions, the emergence and life span of the cyclonic vortex visible in the south corner of the NO WEC solution in Fig. 4 and to identify it in the WEC solution during event 1 . We use a technique that takes advantage of this property in section 5a.

When both wind and waves are large, a previously unresolved type of flow structure arises with WEC. It takes the form of overturning roll cells with thin stripes of positive and negative vorticity $\zeta$, in the areas covered by the propagation direction of the waves (mainly from the northwest in that time period), e.g., as visible during event 2 in Fig. 3. The wave-sheltered areas such as the Santa Barbara Channel do not develop such structure for instance. These roll cells disrupt and replace the classical submesoscale structures when wind and wave amplitudes are strong enough. They do not sit in a particular position as regards preexisting submesoscale structures, but develop widely in relation to the wind and waves, as opposed to submesoscale structures which lie mostly on the edges of mesoscale eddies. To our knowledge, these roll cells have never been resolved in multiscale realistic oceanic simulations.

Defining a roll cell as an elongated lobe of cyclonic vertical vorticity alongside an elongated lobe of anticyclonic vorticity, similar to a small and spatial repeating filament, we estimate an averaged cell width of $L \approx 1.8 \mathrm{~km}$ at the $\mathrm{L} 2$ level and $0.6 \mathrm{~km}$ at the $\mathrm{L} 3$ level. The factor of 3 comes from the immediate reduction of scales from L2 to L3, meaning that the size is resolution sensitive. This leads to the inference that it is underresolved at the L2 level and that an even higher horizontal resolution analysis is needed for confirmation that L3 suffices to describe them properly, but we leave this for a future study.

These structures are predominantly present during event 2 , and strong wind seems to be primarily responsible for their appearance. But, by comparing WEC to NO WEC solutions, it is also clear that WEC enhances them. WEC seems to be related to these structures to the extent that during event 1 , roll cells are only visible in some places of WEC solutions. They arise mainly in anticyclonic regions as shown in Fig. 3 during event 1 for instance. They have a time evolution and development that is correlated to the wind and wave forcings. Once developed, they are advected by larger-scale flows. We further examine these roll-cell structures in section 5 b.

To further analyze the dynamical effect of WEC, we compute the surface kinetic energy spectrum of the solutions over the two large-wave-amplitude events in Fig. 5. The L3 solution contains more energy than L2. This difference reflects the better resolved submesoscale dynamics at higher resolution (Capet et al. 2008a). WEC also increases the energy, particularly at the L3 level. During event 1 the kinetic energy is enhanced at every scale by a factor of at least 2 . This is consistent with the measure of enhanced velocity gradients near the surface. At the largest wavelengths, this is because the better models resolve the submesoscale regime (L3 compared to L2) the more energetic the mesoscale regime (Capet et al. 2008a,b,c), due to the inverse energy cascade (Kraichnan 1967; Charney 1971).

During event 2 the largest flow scales are almost unchanged, but small scales are very energized by WEC. Between length scales $L$ of $200 \mathrm{~m}$ to $2 \mathrm{~km}$, the solution with WEC energy is almost an order of magnitude more than NO WEC. This is the signature of the appearance of the roll cells visible in Figs. 3 and 4, as their estimated size, $L \approx 600 \mathrm{~m}$, lies within this range; the energy spectrum of the WEC L3 solution is peaking very close to this length scale. Similarly, the energy spectrum of the WEC L2 solution has a kink close to the length scale $L \approx 1.8 \mathrm{~km}$. By comparing NO WEC solutions during event 1 and 2 , we can see that the wind itself impacts the shape of the distribution. During the high wind event 2 the large scales are less energized than during event 1 while small scales are more energized, regardless of the inclusion of the waves.

A probability density function (PDF) of near-surface density gradients (Fig. 6) during events 1 and 2 additionally shows WEC effects on submesoscale fronts and filaments. At both L2 and L3 levels (L2 is not shown here for brevity), the PDF of event 2 solutions with and without waves contain fewer high density gradient structures, by an order of magnitude, than the solutions from event 1 . With WEC the tail of the PDF is 


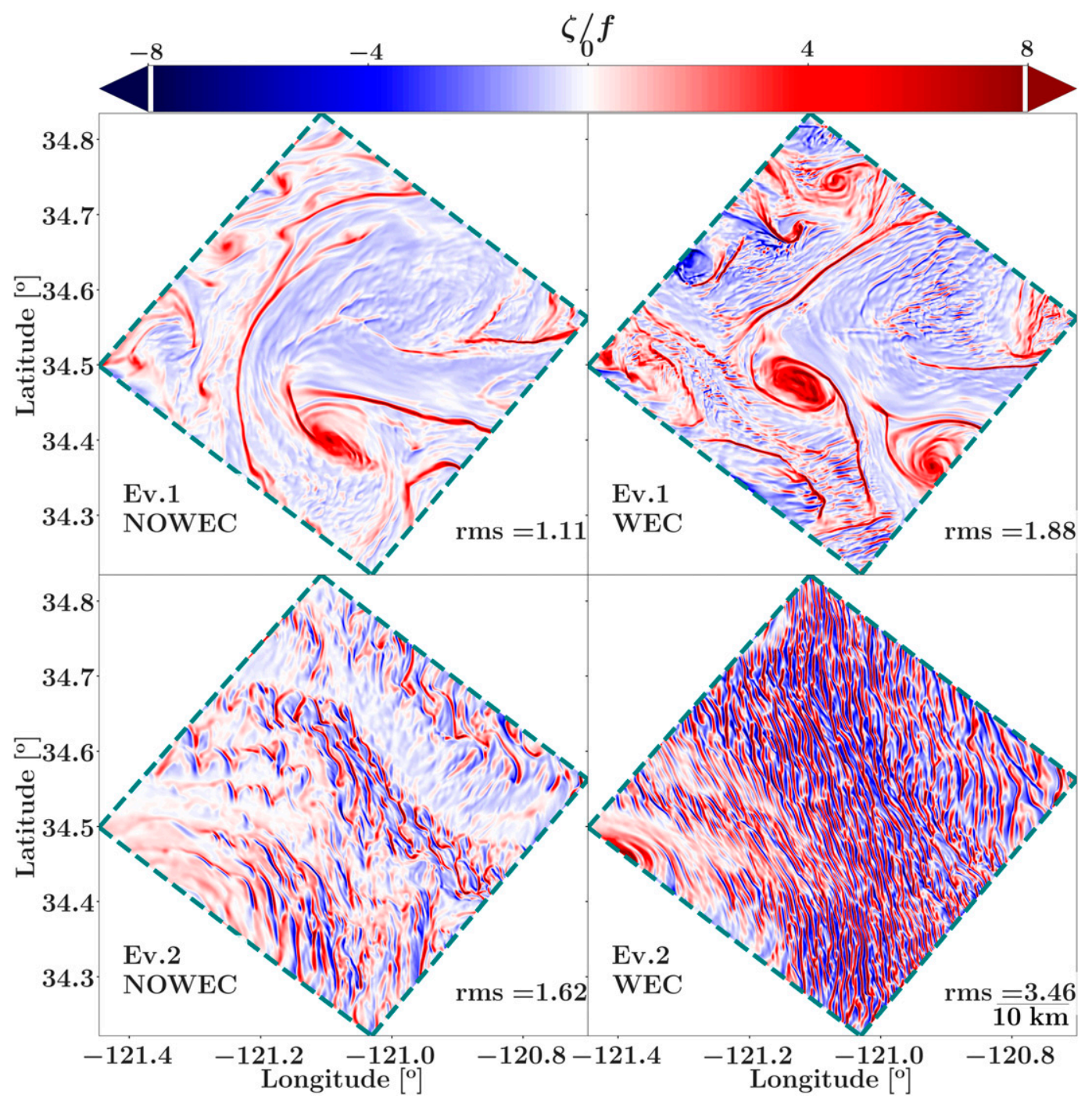

FIG. 4. Snapshots of surface normalized vorticity in the sampled box in the L3 domain at 1600 local time 12 Dec 2006 (event 1) and 1600 local time 28 Dec 2006 (event 2). (left) Solutions without wave forcing and (right) solutions with WEC. Regions of cyclonic vorticity are in red (anticyclonic regions are in blue).

decreased by an extra order of magnitude from NO WEC to WEC solutions. This is consistent with the results of Sun et al. (2020) suggesting that wind can destroy density gradients. It also suggests that the roll cells arising when the wind strengthens, visible in Figs. 3 and 4, replace the preexisting high density submesoscale structures in some locations.

Remotely generated waves (event 1 ) are associated with a slight increase of high density gradient structures compared to the NO WEC solution, particularly visible at L3 (coral curves in Fig. 6). This is consistent with WEC-enhanced horizontally averaged density gradients shown in Fig. 2 near the surface.

Generally, these statistical analyses demonstrate that when $A_{\text {wave }}$ is large, WEC enhances surface density and velocity gradients in the submesoscale regime. During event 1 WEC strengthens submesoscale structures and enlarges their cyclonic vorticity and convergence preference, whereas during event 2 there is a similar statistical increase in near-surface density and velocity gradients. However, we observe the emergence of a previously unresolved type of small-scale roll-cell structures that seems to rarefy high density gradient preexisting submesoscale structures. In this manner, event 2 statistics capture two different mechanisms. Where submesoscale structures are maintained, their velocity and density gradients are increased. But when they are replaced by roll cells, the density gradients are diminished while only velocity gradients are enhanced, because the roll cells' signature is strong, spatially oscillatory vorticity, divergence, and vertical velocity, but one associated with a small horizontal density gradient.

\section{WEC dynamics}

In this section we analyze the statistical properties of multiple dynamical balance equations, to complement the section $3 \mathrm{~b}$ statistical measures of WEC. 

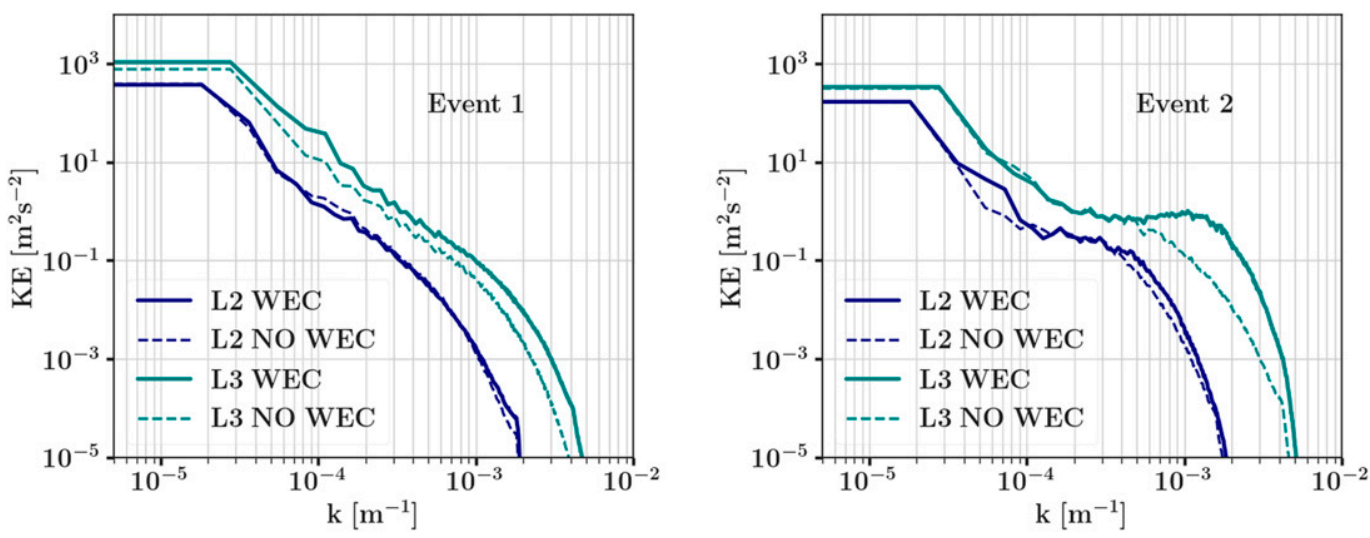

FIG. 5. Time-averaged surface kinetic energy horizontal wavenumber spectra during the two high-wave events. We compare solutions with and without WEC (respectively, solid lines and dashed lines) at the two resolutions (L2 in blue, L3 in teal). We list the RMS values for $\zeta^{2}$ and $\left|\nabla^{h} b\right|^{2}$ in Table 1 and for $\delta$ and $\left|\mathbf{v}_{z}^{h}\right|^{2}$ in Table 2 during the two events for context.

\section{a. Enstrophy, buoyancy gradient, and divergence balance equations}

Following the original idea described in Srinivasan et al. (2021), we derive the equation of evolution for the vertical enstrophy, divergence, horizontal enstrophy, and squared buoyancy gradient, but including here WEC terms for the first time. They are directly derived from the primitive equations with WEC (Uchiyama et al. 2010). Additional terms appear through the vortex forces and the Bernouilli head in the momentum and buoyancy gradient equations. The superscript "St" (after Stokes's name) is used to allow easy identification of these terms.

The equation of evolution for the vertical enstrophy with WEC is

$$
\begin{aligned}
\frac{1}{2} \frac{D^{L} \zeta^{2}}{D t}= & \underbrace{-\zeta(\zeta+f) \delta}_{\Omega_{s}}+\underbrace{\zeta\left(u_{z} w_{y}-v_{z} w_{x}\right)}_{\Omega_{t}} \underbrace{-\zeta(\zeta+f) \delta^{\mathrm{St}}}_{\Omega_{s}^{\mathrm{St}}} \\
& +\underbrace{\zeta\left(u_{z} w_{y}^{\mathrm{St}}-v_{z} w_{x}^{\mathrm{St}}\right)}_{\Omega_{t}^{\mathrm{St}}}+\Omega_{\mathrm{mix}}+\Omega_{\mathrm{diss}},
\end{aligned}
$$

where $D^{L} / D t$ is the material derivative using the Lagrangian velocity as the advective velocity, namely $D^{L} / D t=\partial_{t}+\mathbf{v}^{L} \cdot \nabla=\partial_{t}+\left(\mathbf{v}+\mathbf{v}^{\mathrm{St}}\right) \cdot \nabla$. The terms $\Omega_{s}$ and $\Omega_{t}$ are, respectively, the vortex stretching and vortex tilting terms. Through the vortex force WEC terms appear as their equivalents, $\Omega_{s}^{\mathrm{St}}$ and $\Omega_{t}^{\mathrm{St}}$, containing horizontal gradients of Stokes drift, e.g., $\delta^{\mathrm{St}}=u_{x}^{\mathrm{St}}+v_{y}^{\mathrm{St}}, w_{y}^{\mathrm{St}}$, and $w_{x}^{\mathrm{St}}$.

The evolution equation for divergence with WEC is derived using the Barkan et al. (2019) notation:

$$
\begin{aligned}
\frac{D^{L} \delta}{D t}= & \underbrace{-\delta^{2}+2 J(u, v)}_{\mathscr{F}_{\text {hor }}} \underbrace{+f \zeta}_{\mathscr{F}_{\mathrm{Cor}}} \underbrace{\underbrace{-\nabla^{h 2} \phi^{c}}_{\mathscr{F}_{\mathrm{pres}}}}_{\mathscr{F}_{\text {vert }}-\left(w_{x} u_{z}+w_{y} v_{z}\right)} \\
& \underbrace{+\zeta^{\mathrm{St}}+\mathbf{v}^{h \mathrm{St}} \cdot\left(\nabla_{\perp}^{2}\right) \mathbf{v}^{h}}_{\begin{array}{c}
\text { St } \\
\text { hor }
\end{array}} \underbrace{+f \zeta^{\mathrm{St}}}_{\mathscr{F}_{\mathrm{Cor}}^{\mathrm{St}}} \underbrace{-\left(w_{x}^{\mathrm{St}} u_{z}+w_{y}^{\mathrm{St}} v_{z}\right)}_{\mathscr{F}_{\text {vert }}^{\mathrm{St}}} \\
& +\mathscr{F}_{\text {mix }}+\mathscr{F}_{\text {diss }},
\end{aligned}
$$

where $J$ is the Jacobian operator, $\nabla^{h 2}=\left(\partial_{x x}+\partial_{y y}\right)$ in the horizontal Laplacian operator, and the pressure is a current-wave composite, $\phi^{c}=p+\mathscr{K}$, including $\mathscr{K}$ the Bernouilli head. It is expressed as

$$
\phi^{c}=g\left(\zeta^{c}-\hat{\zeta}\right)-(\mathscr{P}-\mathscr{K})_{\zeta^{c}}+\int(b-K) d z .
$$

The composite sea surface height, $\zeta^{c}=\zeta+\hat{\zeta}$, contains the wave setup $\hat{\zeta}$, which tends to zero away from the shore. The term $-(\mathscr{P}-\mathscr{K})_{\zeta^{c}}$ is the integration constant that represents the wave-induced pressure correction at the surface [see Eqs. (5) and (9) in Uchiyama et al. (2010) for full expressions], and $K$ is the vertical vortex force,

$$
K=\mathbf{v}^{h} \cdot \mathbf{v}_{z}
$$

Other WEC terms are the Stokes-Coriolis term $\mathscr{F}_{\text {Cor }}^{\mathrm{St}}$, the Stokes vertical advective term $\mathscr{F}_{\text {vert }}^{\mathrm{St}}$, and the Stokes horizontal advective term $\mathscr{F}_{\text {hor }}^{\mathrm{St}}$, which is the only one whose form is not

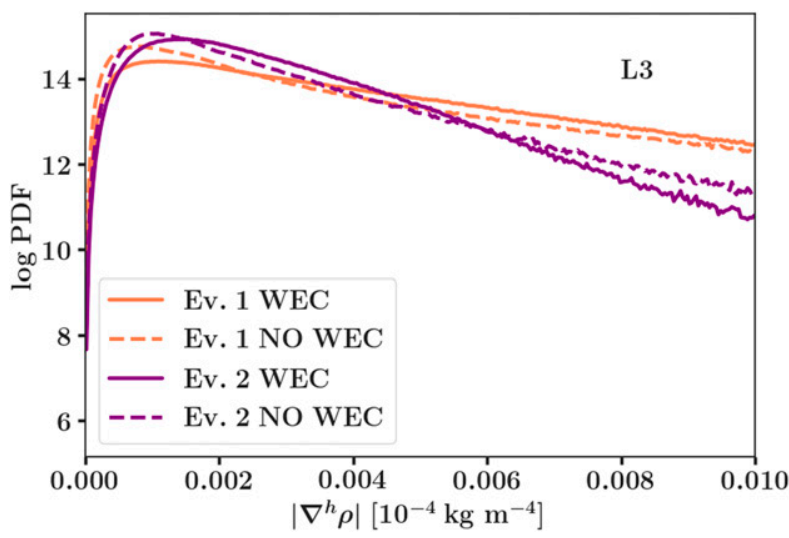

FIG. 6. PDF of horizontal density gradient in L3 during the two large-wave-amplitude events: event 1 in coral and event 2 in purple. 
similar to its NO WEC counterpart $\mathscr{F}_{\text {hor }}$ It contains the product of Eulerian and Stokes vorticities, and the inner product of Stokes drift with the second derivative of horizontal Eulerian velocities. Note that, contrary to the other quantities (vertical enstrophy, horizontal enstrophy, and squared buoyancy gradient), divergence is not a quadratic quantity.

The evolution equation for the horizontal enstrophy or squared vertical shear with WEC is

$$
\begin{aligned}
\frac{1}{2} \frac{D\left|\mathbf{v}_{z}^{h}\right|^{2}}{D t}= & \underbrace{-\left(u_{z}^{2} u_{x}+v_{z}^{2} v_{y}\right)-u_{z} v_{z}\left(v_{x}+u_{y}\right)}_{\Lambda_{h}} \underbrace{+\left|\mathbf{v}_{z}^{h}\right|^{2} \delta}_{\Lambda_{v}} \underbrace{-\left(b_{x} u_{z}+b_{y} v_{z}\right)}_{\Lambda_{b}} \underbrace{-\left(u_{z}^{2} u_{x}^{\mathrm{St}}+v_{z}^{2} v_{y}^{\mathrm{St}}\right)-u_{z} v_{z}\left(v_{x}^{\mathrm{St}}+u_{y}^{\mathrm{St}}\right)}_{\Lambda_{h}^{\mathrm{St}}} \\
& \underbrace{+\left|\mathbf{v}_{z}^{h}\right|^{2} \delta^{\mathrm{St}}}_{\Lambda_{v}^{\mathrm{St}}} \underbrace{-(\zeta+f)\left(u_{z}^{\mathrm{St}} v_{z}-v_{z}^{\mathrm{St}} u_{z}\right)}_{\Lambda^{\mathrm{St}}}+\Lambda_{\text {mix }}+\Lambda_{\text {diss }} .
\end{aligned}
$$

We recover terms as in a case without wave forcing: the vertical and horizontal advective contributions, $\Lambda_{v}$ and $\Lambda_{h}$, and the buoyancy contribution $\Lambda_{b}$. WEC adds vertical and horizontal advective counterparts, $\Lambda_{v}^{\mathrm{St}}$ and $\Lambda_{h}^{\mathrm{St}}$, and a term that contains the vertical shear of Stokes drift $\Lambda^{\mathrm{St}}$.
Finally, we derive the evolution equation of the squared horizontal buoyancy gradient with WEC (also called the Lagrangian frontogenetic tendency equation):

$$
\begin{aligned}
\frac{1}{2} \frac{D^{L}\left|\nabla^{h} b\right|^{2}}{D t}= & \underbrace{-\left(u_{x} b_{x}^{2}+v_{y} b_{y}^{2}\right)-b_{x} b_{y}\left(v_{x}+u_{y}\right)}_{B_{\mathrm{hor}}} \underbrace{-b_{z}\left(b_{x} w_{x}+b_{y} w_{y}\right)}_{B_{\text {vert }}} \underbrace{-\left(u_{x}^{\mathrm{St}} b_{x}^{2}+v_{y}^{\mathrm{St}} b_{y}^{2}\right)-b_{x} b_{y}\left(v_{x}^{\mathrm{St}}+u_{y}^{\mathrm{St}}\right)}_{B_{\text {hor }}^{\mathrm{St}}} \\
& \underbrace{-b_{z}\left(b_{x} w_{x}^{\mathrm{St}}+b_{y} w_{y}^{\mathrm{St}}\right)}_{B_{\text {vert }}^{\mathrm{St}}}+B_{\text {mix }}+B_{\text {diss }} .
\end{aligned}
$$

The buoyancy is defined as $b=-g \rho / \rho_{0}$, with $g$ the gravity acceleration and where the density $\rho$ is normalized by a reference density. Similar to the previous balances, the Stokes drift appears in additional horizontal and vertical advective terms in the frontogenetic tendency equation.

We account for nonconservative forces in all of (1), (2), (5), and (6) through the terms with the subscripts "diss" and "mix." The latter stands for dissipation processes induced by lateral hyperdiffusion from the advection schemes for tracers and momenta, and the former for the parameterized vertical mixing. The hyperdiffusion can be estimated by the difference between the third-order, upstream-biased, and fourth-order central advection schemes (Lemarié et al. 2012).

\section{b. WEC impacts on dynamical balances}

The term magnitudes in the three quadratic balance equations, (1), (5), and (6), and in the divergence Eq. (2) are listed in Tables 1 and 2, where the root-mean-square (RMS) operation is performed within the open-ocean dashed box of L3 (see Fig. 1) at $1 \mathrm{~m}$ below the surface during both high-wave events. This allows highlighting the terms which contribute most to the balances. For the squared buoyancy gradient, we do not provide an offline estimate of the $B_{\text {diss }}$ RMS value, because this (hyper)diffusion is implicit in the advection operator in ROMS, but we expect this term to be always frontolytic, e.g., as in Gula et al. (2014).

Event 1 without waves (first column of Tables 1 and 2) illustrates familiar submesoscale dynamics. The vertical enstrophy evolution is controlled at leading order by the balance of vortex stretching and nonconservative terms. The divergence rate mainly reflects advective processes. Both the squared vertical shear and buoyancy gradient evolutions are substantially influenced by vertical mixing. The comparison between the two events without waves (first and third column of Tables 1 and 2) shows the action of the onset of strong wind: vertical mixing terms amplify, and surface current intensification leads to larger surface nonlinearities, larger vertical shear, and smaller buoyancy gradients.

The WEC impact on these balances appears in two ways: indirect (Table 1) and direct (Table 2). The indirect impact of WEC occurs in the vertical enstrophy and squared buoyancy gradient balances as indicated in the second and fourth columns of Table 1 where waves are included. The RMS values of these two fields are enhanced by an order of magnitude in both high-wave events compared to their respective cases without waves. Consistently, the RMS value of terms that contain velocity gradients $\left(\Omega_{s}, \Omega_{t}, B_{\text {hor }}\right.$, and $B_{\text {vert }}$, for instance) increases, while they do not explicitly depend on Stokes drift or other WEC variables. These advective terms are the most important ones in these balances when WEC is included, and the WEC terms are actually negligible in these two balances because the Stokes drift is generally horizontally smooth (i.e., horizontal gradients of Stokes drift are small and therefore terms such as $\Omega_{s}^{\mathrm{St}}, \Omega_{t}^{\mathrm{St}}, B_{\mathrm{hor}}^{\mathrm{St}}$, and $B_{\mathrm{vert}}^{\mathrm{St}}$ are negligible). Hence, WEC enhances velocity gradients fields, which in turn leads to larger sources of both vertical enstrophy and squared buoyancy gradient. 
TABLE 1. Vertical enstrophy [Eq. (1)] and squared buoyancy gradient [Eq. (6)]: RMS values at $1 \mathrm{~m}$ depth within the L3 open-ocean sampling domain.

\begin{tabular}{|c|c|c|c|c|}
\hline WEC included & No & Yes & No & Yes \\
\hline Event No. & 1 & 1 & 2 & 2 \\
\hline \multicolumn{5}{|l|}{ Vertical enstrophy } \\
\hline$\zeta^{2}\left(\mathrm{~s}^{-2}\right)$ & $2.7 \times 10^{-8}$ & $1.6 \times 10^{-7}$ & $3.4 \times 10^{-8}$ & $1.4 \times 10^{-7}$ \\
\hline$\Omega_{s}\left(\mathrm{~s}^{-3}\right)$ & $1.1 \times 10^{-11}$ & $3.6 \times 10^{-10}$ & $8.9 \times 10^{-12}$ & $1.0 \times 10^{-10}$ \\
\hline$\Omega_{t}\left(\mathrm{~s}^{-3}\right)$ & $8.1 \times 10^{-13}$ & $6.5 \times 10^{-12}$ & $1.8 \times 10^{-12}$ & $2.7 \times 10^{-11}$ \\
\hline$\Omega_{s}^{\mathrm{St}}\left(\mathrm{s}^{-3}\right)$ & & $1.5 \times 10^{-13}$ & & $4.7 \times 10^{-13}$ \\
\hline$\Omega_{t}^{\mathrm{St}}\left(\mathrm{s}^{-3}\right)$ & & $3.7 \times 10^{-13}$ & & $1.9 \times 10^{-12}$ \\
\hline$\Omega_{\text {mix }}\left(\mathrm{s}^{-3}\right)$ & $1.3 \times 10^{-12}$ & $2.3 \times 10^{-11}$ & $4.5 \times 10^{-12}$ & $3.6 \times 10^{-11}$ \\
\hline$\Omega_{\text {diss }}\left(\mathrm{s}^{-3}\right)$ & $1.4 \times 10^{-12}$ & $4.1 \times 10^{-11}$ & $2.3 \times 10^{-12}$ & $2.5 \times 10^{-11}$ \\
\hline \multicolumn{5}{|c|}{ Squared buoyancy gradient } \\
\hline$\left(\nabla^{h} b\right)^{2}\left(\mathrm{~s}^{-4}\right)$ & $4.3 \times 10^{-13}$ & $1.0 \times 10^{-12}$ & $1.5 \times 10^{-13}$ & $4.0 \times 10^{-12}$ \\
\hline$B_{\text {hor }}\left(\mathrm{s}^{-5}\right)$ & $9.2 \times 10^{-17}$ & $9.3 \times 10^{-16}$ & $4.3 \times 10^{-17}$ & $1.6 \times 10^{-15}$ \\
\hline$B_{\text {vert }}\left(\mathrm{s}^{-5}\right)$ & $4.4 \times 10^{-17}$ & $1.7 \times 10^{-16}$ & $1.7 \times 10^{-18}$ & $1.1 \times 10^{-15}$ \\
\hline$B_{\text {hor }}^{\mathrm{St}}\left(\mathrm{s}^{-5}\right)$ & & $3.5 \times 10^{-19}$ & & $2.3 \times 10^{-18}$ \\
\hline$B_{\mathrm{vert}}^{\mathrm{St}}\left(\mathrm{s}^{-5}\right)$ & & $1.3 \times 10^{-17}$ & & $5.4 \times 10^{-18}$ \\
\hline$B_{\text {mix }}\left(\mathrm{s}^{-5}\right)$ & $2.4 \times 10^{-16}$ & $6.4 \times 10^{-16}$ & $5.2 \times 10^{-17}$ & $1.0 \times 10^{-15}$ \\
\hline
\end{tabular}

The WEC impact is direct when the WEC terms are of leading-order magnitude (Table 2). For instance, the Stokes horizontal advective term $\mathscr{F}_{\text {hor }}^{\text {St }}$ is strong in the divergence balance. However, as previously stated, this latter actually reflects the enhanced gradients of the Eulerian fields with WEC (the dominant part of this term comes from the second derivative of the Eulerian field). But, in the vertical shear balance the term $\Lambda^{\text {St }}$ is large, and this comes from the vertical shear of the Stokes drift itself. These terms induce explicit, nonnegligible additions to the dynamical balances, and they are a starting point to understand the modifications these analyzed quantities undergo due to WEC.

\section{c. Vertical structure}

Here, we further assess WEC influence on the dynamical balances by examining vertical profiles of contributing terms. We perform a conditional horizontal averaging by isolating regions of high normalized surface cyclonic vorticity (i.e., $\zeta / f>$ 2 ). This condition aims at capturing submesoscale front and filament signals statistically. Figure 7 shows the resulting vertical structure of the dominant terms contributing to the

TABLE 2. Horizontal enstrophy [Eq. (5)] and divergence [Eq. (2)]: RMS values at $1 \mathrm{~m}$ depth within the L3 open-ocean sampling domain.

\begin{tabular}{|c|c|c|c|c|}
\hline WEC included & No & Yes & No & Yes \\
\hline Event No. & 1 & 1 & 2 & 2 \\
\hline \multicolumn{5}{|c|}{ Horizontal enstrophy } \\
\hline$\left|\mathbf{v}_{z}^{h}\right|^{2}\left(\mathrm{~s}^{-3}\right)$ & $2.5 \times 10^{-4}$ & $2.4 \times 10^{-4}$ & $5.3 \times 10^{-4}$ & $5.7 \times 10^{-4}$ \\
\hline$\Lambda_{h}\left(\mathrm{~s}^{-3}\right)$ & $1.1 \times 10^{-8}$ & $2.7 \times 10^{-8}$ & $1.7 \times 10^{-1}$ & $7.2 \times 10^{-8}$ \\
\hline$\Lambda_{v}\left(\mathrm{~s}^{-3}\right)$ & $1.7 \times 10^{-8}$ & $6.4 \times 10^{-8}$ & $2.8 \times 10^{-8}$ & $1.5 \times 10^{-7}$ \\
\hline$\Lambda_{b}\left(\mathrm{~s}^{-3}\right)$ & $3.0 \times 10^{-9}$ & $3.9 \times 10^{-9}$ & $2.5 \times 10^{-9}$ & $6.8 \times 10^{-9}$ \\
\hline$\Lambda_{h}^{\mathrm{St}}\left(\mathrm{s}^{-3}\right)$ & & $1.5 \times 10^{-10}$ & & $1.4 \times 10^{-9}$ \\
\hline$\Lambda_{v}^{\mathrm{St}}\left(\mathrm{s}^{-3}\right)$ & & $2.9 \times 10^{-10}$ & & $2.2 \times 10^{-9}$ \\
\hline$\Lambda^{\mathrm{St}}\left(\mathrm{s}^{-3}\right)$ & & $1.3 \times 10^{-8}$ & & $2.7 \times 10^{-8}$ \\
\hline$\Lambda_{\operatorname{mix}}\left(\mathrm{s}^{-3}\right)$ & $4.2 \times 10^{-8}$ & $9.5 \times 10^{-8}$ & $8.3 \times 10^{-8}$ & $1.5 \times 10^{-7}$ \\
\hline$\Lambda_{\text {diss }}\left(\mathrm{s}^{-3}\right)$ & $8.6 \times 10^{-10}$ & $7.5 \times 10^{-9}$ & $9.6 \times 10^{-10}$ & $5.4 \times 10^{-9}$ \\
\hline \multicolumn{5}{|l|}{ Divergence } \\
\hline$\delta\left(\mathrm{s}^{-1}\right)$ & $5.2 \times 10^{-5}$ & $1.3 \times 10^{-4}$ & $5.9 \times 10^{-5}$ & $2.1 \times 10^{-4}$ \\
\hline $\mathscr{F}_{\text {hor }}\left(\mathrm{s}^{-2}\right)$ & $1.3 \times 10^{-8}$ & $1.4 \times 10^{-7}$ & $1.6 \times 10^{-8}$ & $1.1 \times 10^{-7}$ \\
\hline $\mathscr{F}_{\mathrm{Cor}}\left(\mathrm{s}^{-2}\right)$ & $6.3 \times 10^{-9}$ & $1.1 \times 10^{-8}$ & $7.9 \times 10^{-9}$ & $2.0 \times 10^{-8}$ \\
\hline $\mathscr{F}_{\text {pres }}\left(\mathrm{s}^{-2}\right)$ & $1.5 \times 10^{-9}$ & $1.3 \times 10^{-8}$ & $9.3 \times 10^{-10}$ & $2.4 \times 10^{-8}$ \\
\hline $\mathscr{F}_{\text {vert }}\left(\mathrm{s}^{-2}\right)$ & $3.6 \times 10^{-9}$ & $1.2 \times 10^{-8}$ & $3.7 \times 10^{-9}$ & $1.7 \times 10^{-8}$ \\
\hline $\mathscr{F}_{\text {hor }}^{\mathrm{St}}\left(\mathrm{s}^{-2}\right)$ & & $7.1 \times 10^{-8}$ & & $5.4 \times 10^{-7}$ \\
\hline $\mathscr{F}_{\mathrm{Cor}}^{\mathrm{St}}\left(\mathrm{s}^{-2}\right)$ & & $8.0 \times 10^{-11}$ & & $1.5 \times 10^{-10}$ \\
\hline $\mathscr{F}_{\text {vert }}^{\mathrm{St}}\left(\mathrm{s}^{-2}\right)$ & & $3.1 \times 10^{-9}$ & & $1.6 \times 10^{-8}$ \\
\hline $\mathscr{F}_{\text {mix }}\left(\mathrm{s}^{-2}\right)$ & $5.6 \times 10^{-9}$ & $2.1 \times 10^{-8}$ & $1.9 \times 10^{-8}$ & $1.9 \times 10^{-7}$ \\
\hline $\mathscr{F}_{\text {diss }}\left(\mathrm{s}^{-2}\right)$ & $3.9 \times 10^{-9}$ & $3.4 \times 10^{-8}$ & $8.9 \times 10^{-9}$ & $8.4 \times 10^{-8}$ \\
\hline
\end{tabular}




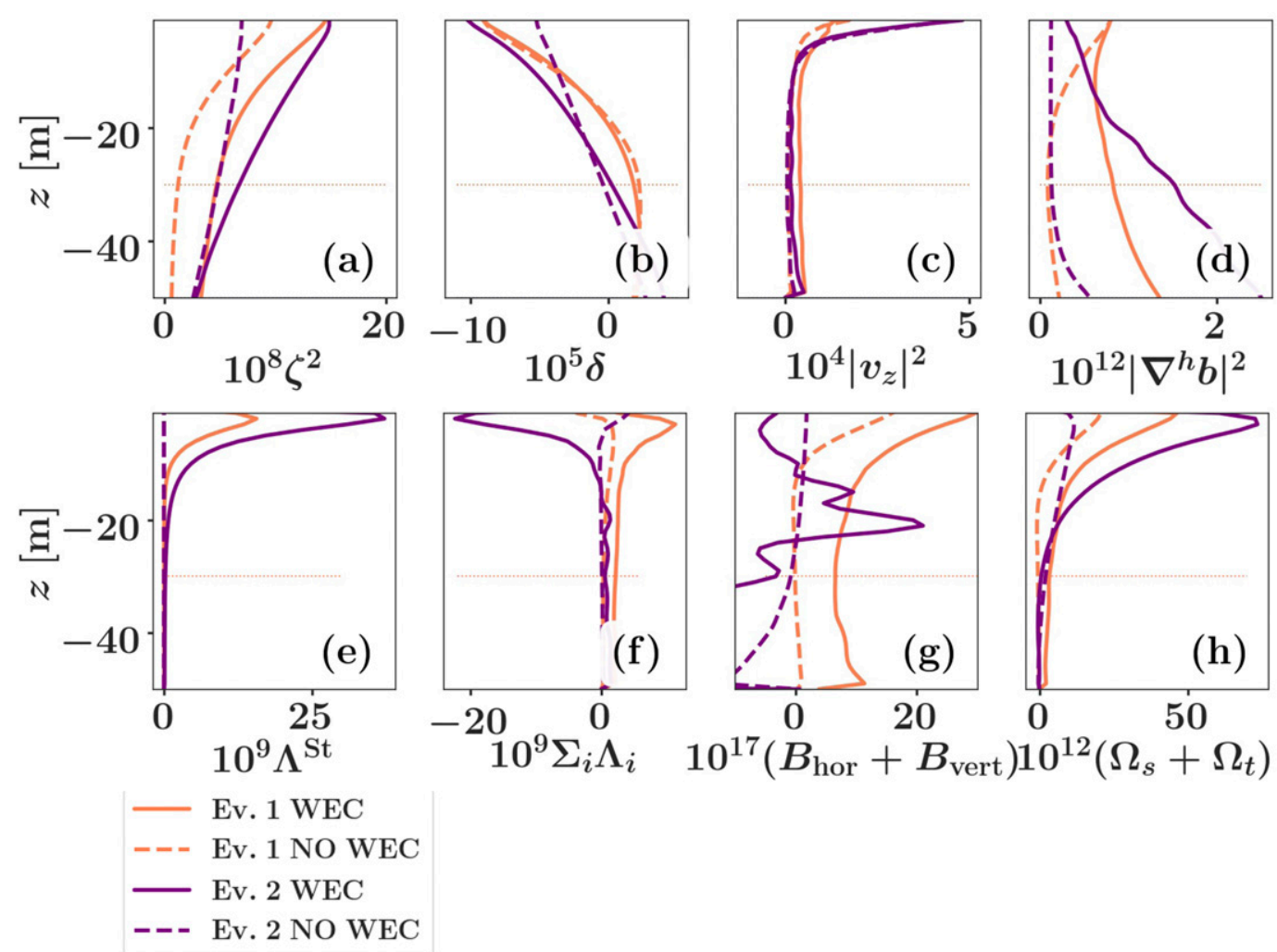

FIG. 7. Conditionally averaged $\left(\zeta / f>2\right.$, see text) vertical structures of (a) vertical enstrophy $\zeta^{2}\left(\mathrm{~s}^{-2}\right)$, (b) divergence $\delta\left(\mathrm{s}^{-1}\right)$, (c) horizontal enstrophy $\left|\mathbf{v}_{z}^{h}\right|^{2}\left(\mathrm{~s}^{-2}\right)$, and (d) squared buoyancy gradient $\left|\nabla^{h} b\right|^{2}\left(\mathrm{~s}^{-4}\right)$ and (e)-(h) dominant terms contributing to their dynamical balances during both large-wave-amplitude events (coral for event 1, purple for event 2). Solutions without WEC are dashed lines, and solid lines include WEC. Terms of the horizontal enstrophy balance (5) have been gathered such as $\Sigma_{i} \Lambda_{i}=\Lambda_{h}+\Lambda_{v}+\Lambda_{b}\left(\mathrm{~s}^{-3}\right)$ in (f). Panel (e) shows the term $\Lambda_{\mathrm{St}}\left(\mathrm{s}^{-3}\right)$ also from (5). Panel (g) shows the sum $B_{\text {hor }}+B_{\text {vert }}\left(\mathrm{s}^{-5}\right)$ from (6), and panel (h) the sum $\Omega_{t}+\Omega_{s}\left(\mathrm{~s}^{-3}\right)$ from (1). The time-averaged mixed layer depth of event 1 is represented with orange dotted lines. During event 2 , the mixed layer extends approximately down to $50 \mathrm{~m}$ deep. We only show the L3 solutions here. Quantities vanish below $\simeq 50 \mathrm{~m}$ depth.

balances of vertical enstrophy, divergence, horizontal enstrophy, and squared buoyancy gradient during both high-wave events. The main signal of horizontal enstrophy is confined to the first $10 \mathrm{~m}$ (Figs. 7c,e,f), which is comparable to the decay length of the Stokes drift, as opposed to the $30 \mathrm{~m}$ vertically averaged extension of the signals from other balances corresponding to the average mixed layer depth during event 1 (Figs. 7a,b,d,g,h). During event 2, the profiles and the mixed layer are deeper due to the strong wind, reaching on average $50-60 \mathrm{~m}$ in depth. Beside stratification, all the profiles vanish rapidly below this depth.

As illustrated in the first row of Fig. 7, the conditional averaging successfully captures high vorticity features (Fig. 7a) associated with surface convergence (Fig. 7b) and high surface buoyancy gradients (Fig. 7d). But, surface buoyancy gradients from event 2 are on average smaller than during event 1, corroborating the similar result in Fig. 6, which shows that the combination of high wind and waves does not favor large density gradients.

Note that, because usually extrema of vorticity do not coincide with extrema of divergence, the divergence balance is less trusted as properly reflecting submesoscale dynamics when using this conditional averaging on high vorticity.

\section{1) EVEnt 1: REMOTE SWELL}

During event 1 (coral curves, Fig. 7), the extra Stokes-drift vertical-shear term $\Lambda^{\text {St }}$ is of leading order in the horizontal enstrophy evolution (Fig. 7e). Combined with the Eulerian advection sources $\left(\Sigma_{i} \Lambda_{i}\right.$ in Fig. $\left.7 f\right)$, this leads to larger vertical shear at almost all depths when WEC is included (Fig. 7c). In turn, terms that contain the vertical shear, in particular the vortex tilting term $\Omega_{t}$ (Fig. $7 \mathrm{~h}$ ) as a large source of vertical enstrophy, increase with WEC. Additionally, larger surface relative vorticity in WEC leads to larger vortex stretching, which actually dominates as a vorticity source. Ultimately, both vortex stretching and tilting $\Omega_{s}+\Omega_{t}$ (Fig. 7h), are enhanced with WEC as sources. This is the indirect mechanism of WEC by which surface velocity gradients fields are enhanced.

\section{2) EVENT 2: LOCAL WAVE GENERATION}

In the vertical enstrophy balance there is a competition between the combined vortex stretching and tilting, $\Omega_{s}+\Omega_{t}$ in (1) 
(purple curves in Fig. 7h), as sources, and a competition between the nonconservative effects $\Omega_{\text {diss }}+\Omega_{\text {mix }}$ (not shown), as sinks. But, in event 2, source terms peak below the surface. These profiles reveal the dominance of the vortex-tilting term $\left(\Omega_{t}\right)$, where vertical velocity $w$ is largest below the surface (Gula et al. 2014). As shown in Fig. 5, small submesoscale structures have more energy with WEC, and the roll cells, visible in Fig. 4, emerge and are typically associated with high $w_{x}$ and $w_{y}$, and therefore larger vortex tilting during event 2 . This is the mechanism by which wind-generated waves tilt horizontal vorticity anomalies into the vertical. Also, the vertical shear is larger during event 2 (compared to event 1 (Fig. 7c), which further increases the vortex tilting with the location of peak shear generation approximately collocated with maximum vertical enstrophy generation (Figs. 7e,h). In this manner WEC generates more vertical enstrophy during event 2.

Near the surface the advective processes $\left[B_{\text {vert }}, B_{\text {hor }}\right.$ of (6)] are, contrary to event 1 , sinks for the squared buoyancy gradient evolution (Fig. $7 \mathrm{~g}$ ). This reflects the onset of the roll cells that exhibit a spatially oscillatory pattern in vorticity, divergence, and vertical velocity. These structures contain large $w_{x}$ and $w_{y}$ values that can control the magnitude of the $B_{\text {vert }}$ term. A $\zeta / f>2$ conditional averaging isolates $B_{\text {vert }}<0$ terms (not shown) that dominate the full advective term. This dominance by $B_{\text {vert }}$ is distinctive in the wind-forced wave regime and consistently illustrates the weakening of density-gradient structures during that event (Figs. 6 and 7).

To summarize, we find that the vertical shear of the Stokes drift is responsible for the important extra source of vertical current shear $\Lambda_{\mathrm{St}}$, which in turn increases the vortex tilting $\Omega_{t}$ during event 1 and ultimately amplifies submesoscale vorticity near the surface. Under local wind-generated waves, the Eulerian advection (through $B_{\text {vert }}$ ) is often responsible for the destruction of submesoscale structures, as evidenced by the decrease of surface buoyancy gradient. The emergence of roll cells is associated with vertical enstrophy generation, supported by the enhanced vertical shear induced by the Stokes drift vertical shear. This analysis untangles the statistical properties of event 2 that capture both the destruction of classical submesoscale structures (decrease of surface velocity and density gradients) and the emergence of the roll cells (increase solely of surface velocity gradients).

\section{Flow structures}

In this section we examine some individual flow structures to complement the previous statistical and dynamical balance analyses. We compare an individual dense filament in simulations with and without WEC during event 1 which contains a large remotely generated swell. Second, we examine in detail the roll cells that emerge during event 2 , i.e., an event with strong wind and large wind-forced waves.

\section{a. A submesoscale front with remote swell}

We make two integrations (one with WEC and one without) starting two days prior to the time period identified as event 1 , namely, the event of large wave amplitude provoked by a remote swell. From this common initial state the two simulations diverge in time. But, close to initialization it is possible to identify some submesoscale structures with an appreciable time correlation between both simulations. A caveat of this method is that, if we aim at a similar state of the filament, they will look alike but it does not mean that they are at the same stage of their frontogenesis. Another limitation to our analysis approach is that there is a large background flow resulting in a sensitivity to the selection area when performing spatial averaging (e.g., the demarcations of frontal locations in Fig. 9).

In the simulation without WEC, it is expected that the turbulent thermal wind approximate balance (TTW) (McWilliams 2018) would explain to a large degree the properties of a submesoscale front or filament in the open ocean: when vertical mixing is strong, the geostrophic balance does not hold close to the surface, and a horizontal momentum balance can be reached between the pressure gradient, Coriolis, and vertical mixing forces. But as demonstrated in Dauhajre and McWilliams (2018), a strong diurnal cycle can induce a generalization to the transient TTW balance $\left(\mathrm{T}^{3} \mathrm{~W}\right)$ that also includes horizontal acceleration in the balance. For simplicity here, we aim at times when TTW and $\mathrm{T}^{3} \mathrm{~W}$ agree the most, i.e., around 0700 or 1900 local time (seasonal approximate time for the change from day to night and reverse).

In both simulations with and without WEC, at approximately 0700 local time of the third day of event 1 , we follow a filament from its initial state. It exhibits strong convergence and cyclonic vorticity at the surface (Fig. 8). We show the demarcation in both domains in between which we fit an alongfilament polynomial function onto the cyclonic trace of the filament, analogous to Gula et al. (2014). It permits a separation of the flow, by rotating $(x, y)$ into the local along- and cross-filament directions. We display along-filament averages of vorticity and divergence for the filament in both simulations in Fig. 9. The approximately vertically oriented isopycnals near the surface indicate a typical, open-ocean dense filament structure, $\mathscr{O}(100) \mathrm{m}$ in width, that extends vertically through an (50) m surface boundary layer. Both WEC and NO WEC filaments show a strong cyclonic vorticity signal at the frontal location (in between the green dashed vertical lines in Fig. 9). Similarly, both cases exhibit a typical secondary circulation characterized by surface convergence and downwelling. Upwelling is operating sparsely on the sides of filaments, as illustrated by regions of positive divergence in Fig. 9, around $10 \mathrm{~km}$ for the NO WEC case and at $12 \mathrm{~km}$ for the WEC case for instance.

We compute the momentum balance projected in the along and cross-front directions of the filament and show the vertical structure of the dominant terms in Fig. 10 averaged at the frontal location (dashed vertical lines in Fig. 9).

$$
\underbrace{\mathbf{v}_{t}^{h}}_{\text {rate }}+\underbrace{\left(\mathbf{v}^{h} \cdot \nabla^{h}\right) \mathbf{v}^{h}+w \mathbf{v}_{z}^{h}}_{\text {advection }}+\underbrace{f \hat{\mathbf{z}} \wedge \mathbf{v}^{h}}_{\text {Coriolis }}+\underbrace{\nabla^{h} \phi_{c}}_{\text {pressure }}-\underbrace{\mathbf{J}^{h}}_{\mathrm{VF}}-\mathbf{F}=0 .
$$

The term $\mathbf{J}^{h}$ is the horizontal vortex force:

$$
\mathbf{J}^{h}=-\hat{\mathbf{z}} \wedge \mathbf{v}^{h \mathrm{St}}\left[\left(\hat{\mathbf{z}} \cdot \nabla^{h} \wedge \mathbf{v}^{h}\right)+f\right]-w^{\mathrm{St}} \mathbf{v}_{z}^{h}
$$




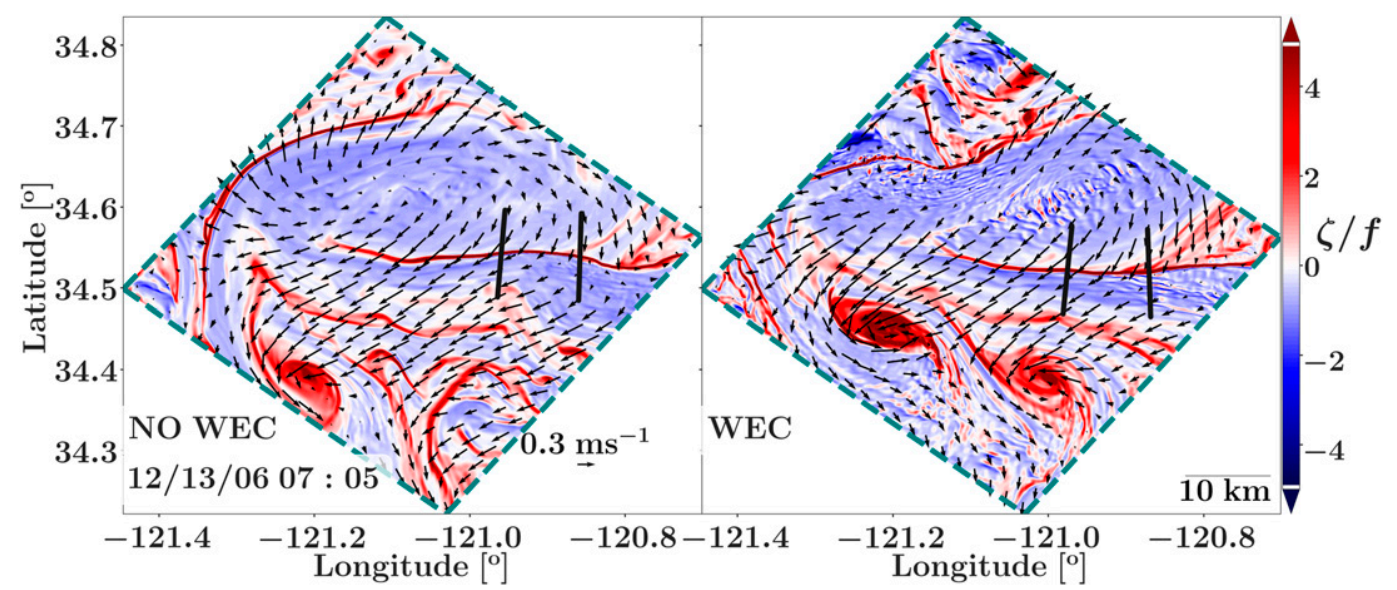

FIG. 8. Snapshot of normalized surface vorticity from the L3 solutions (right) with and (left) without WEC. The time is 0705 local time $13 \mathrm{Dec} 2006$ (which is during event 1). We superimpose surface Lagrangian currents in black. The black lines demarcate the portion of a filament of cyclonic vorticity we identify in both solutions and in between, which we make a polynomial fit allowing us to separate quantities into their along- and cross-front directions.

The nonconservative forces are represented by $\mathbf{F}$, including the vertical mixing term $\partial_{z}\left(K_{v} \partial_{z} \mathbf{v}^{h}\right)$. Terms are computed offline on the left-hand side of the momentum equation. The TTW imbalance residual is very small near the surface in the filament without WEC in both directions: see the black dashed lines in Fig. 10. Interestingly, with WEC the TTW residual departs from zero and the vortex force is significant. A strong alongfront background flow is partly responsible for the departure of residuals from zero as it translates into a large Coriolis force. But, the residual from the summation of TTW with the vortex force (pink dashed lines) tends to be closer to zero at the frontal location. The relatively large magnitude of the vortex force indicates that it significantly influences the local filament circulation, even though the summation of TTW with the vortex force leaves a residual and is therefore an incomplete description of the filament dynamics in this case.

\section{b. Submesoscale roll cells}

Shown in Figs. 3, 4, 11, and 12 is an oscillatory pattern of positive and negative vorticity, associated with large positive and negative divergence and small density gradient (see the third panel of 12). It emerges when strong wind and associated large wind-generated waves arise, most dramatically in the WEC solutions. We provide in Fig. 13 a vertical section of two of these roll cells and show their $3 \mathrm{D}$ velocity field. The vectors show the roll cells circulation is mainly clockwise and there are jets in the along-roll direction (e.g., at $y \sim 6 \mathrm{~km}$ near surface of Fig. 13b).

These roll cells potentially resemble Langmuir cells in LT (Langmuir 1938; Harcourt and D'Asaro 2008; McWilliams et al. 2014; Li and Fox-Kemper 2017). Langmuir cells are counterrotating vortices, arising from a vertical shear instability of the wind-driven (Ekman) currents, first observed with axes nearly aligned with the wind, and created by an instability of the winddriven boundary layer flow by which waves tilt vertical vorticity anomalies into the horizontal (Craik and Leibovich 1976). Conversely, here the observed small-scale structures are roll cells that occur in conjunction with submesoscale dynamics and horizontal buoyancy gradients in the presence of wind and Stokes vertical shear. We discuss here a few of their properties in the L3 solution and compare them to the known properties of Langmuir cells in the literature to provide a first-pass assessment that attempts to categorize these numerically unexplored structures.

\section{c. Langmuir number}

To give some sense of the relative contributions of the wind and wave components in the L3 WEC solution, we follow McWilliams et al. (1997) that defines a turbulent (when the Reynolds number is large) Langmuir number as

$$
\mathrm{La}_{\text {tur }}=\sqrt{\frac{U_{\star}}{U^{\mathrm{St}}}},
$$

where the frictional velocity is $U_{\star}=(\tau / \rho)^{1 / 2}$ and $\tau$ is the wind stress. When this number is below an $\mathscr{O}(1)$ threshold, LT is expected. During event 2, at 1535 local time 27 December 2006 (see Fig. 11), the averaged wind stress is $\tau=0.6 \mathrm{~N} \mathrm{~m}^{-2}$, with an averaged surface Stokes drift amplitude $U^{\mathrm{St}}=0.2 \mathrm{~m} \mathrm{~s}^{-1}$. This implies a turbulent Langmuir number of 0.3 , well within the LT regime.

In the LES performed by Van Roekel et al. (2012), e.g., the Langmuir cells have a lateral size around $20 \mathrm{~m}$. In McWilliams et al. (1997), a typical, yet irregular, spacing between the structures is about $50 \mathrm{~m}$. While in L3, the horizontal spacing of the roll cells is on average $600 \mathrm{~m}$, well above a typical Langmuir cell size. These LES examples have settings (small domain size and moderate wind) that favor small LC and large-aspect ratio structures have been observed and simulated before (Marmorino et al. 2005; Sundermeyer et al. 2014). But here, the manifestation of roll cells in L3 is inherently limited by its horizontal grid resolution of $d x=100 \mathrm{~m}$. LT has its strongest vertical vorticity intensified near the surface on the scale of the Stokes drift profile (McWilliams et al. 1997). The averaged $e$-folding of the Stokes drift during event 2 is around $10 \mathrm{~m}$ 

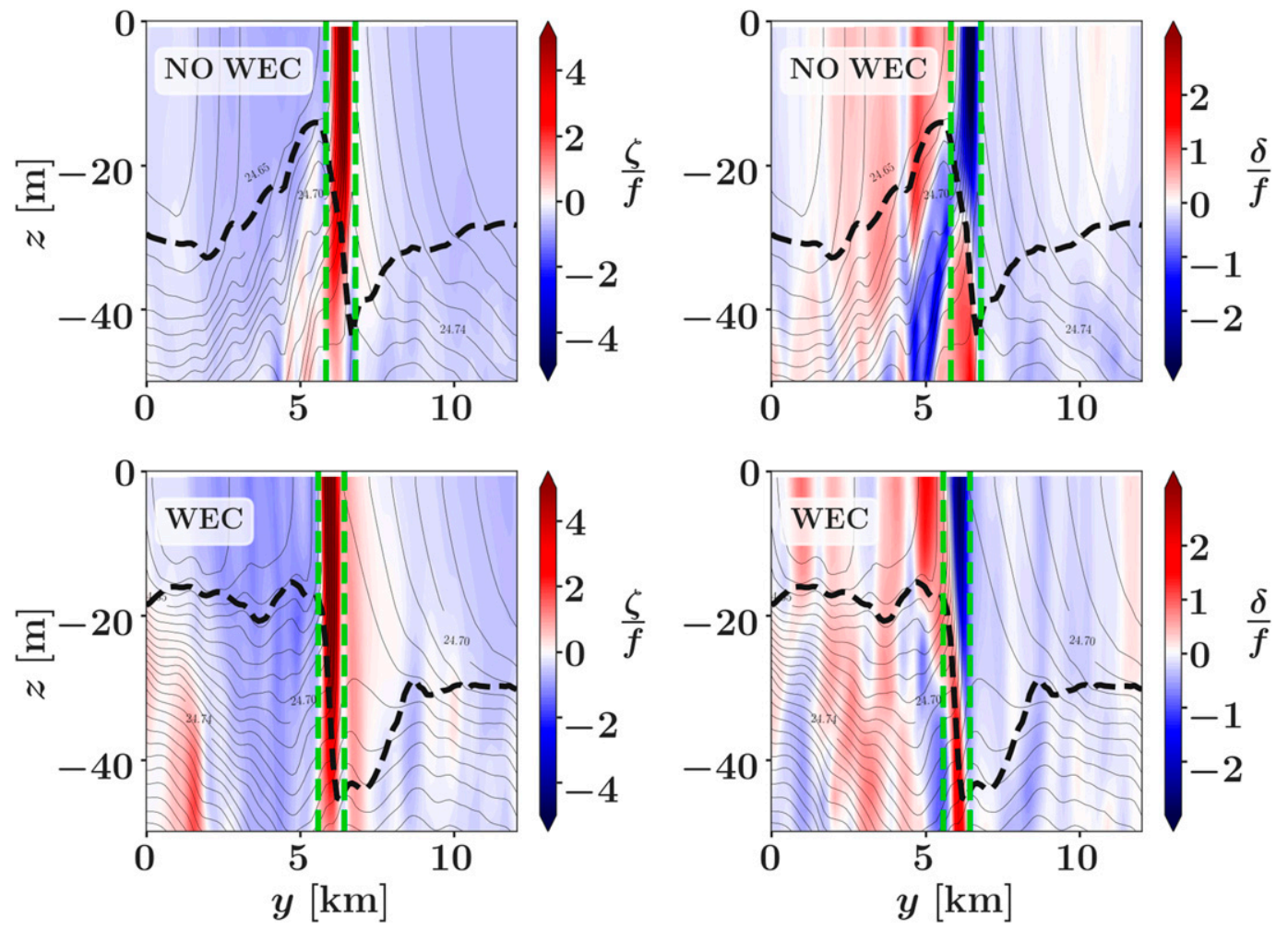

FIG. 9. Cross-front vertical sections of alongfront averaged normalized vorticity and divergence in the portion of filament identified in Fig. 8 in the WEC and NO WEC L3 solutions during event 1. Isopycnals and mixed layer depth (dashed line) are plotted in black. The contour interval of the isopycnals is $0.01 \mathrm{~kg} \mathrm{~m}^{-3}$. A window for averaging near the front location is demarcated in green (averaging-window width $\simeq 1.1 \mathrm{~km}$ ).

depth. As is visible in Fig. 12, the roll cells extend vertically to the entire mixed layer depth, which is around $60 \mathrm{~m}$. The latter scalings (the horizontal spacing and the vertical scale) are not in agreement with typical descriptions of Langmuir cells. Thus, both the roll cells here (and upper-ocean submesoscale currents more generally) and Langmuir cells tend to fill the surface mixed layer. However here, the larger size of the roll cells here may partly be a $d x$ resolution limitation. A finer horizontal resolution would allow smaller structures to emerge with a likely limited vertical expansion (though such an investigation is beyond the scope of this paper).

Another consideration is the orientation of the roll cells. LT usually has its strongest horizontal vorticity aligned longitudinally with the wind and waves (McWilliams et al. 1997). During event 2, in which the wind and wave directions are mostly aligned (pointing ESE, not shown), LT would be expected to align in the same direction (Van Roekel et al. 2012). Meanwhile, as shown on Fig. 11, the roll cells actually align more with the surface Lagrangian current $\left(\mathbf{v}+\mathbf{v}^{h \mathrm{St}}\right)$, which is more in agreement with Gnanadesikan and Weller (1995) who suggest that Langmuir cells align with the direction of maximum Lagrangian velocity shear. This is also reported qualitatively in Sullivan et al. (2012) for hurricane conditions with LES solutions forced with realistic Stokes drift profiles from WW3. Ultimately, this shows that in L3 the Eulerian currents are strong and dominate the roll-cell orientation.

\section{d. Eddy kinetic energy conversion}

Finally, we consider the eddy kinetic energy (EKE) balance. Without waves the most important conversion terms in the EKE balance are

$$
\begin{aligned}
\mathrm{HRS}+\mathrm{VRS}= & -\left\langle u^{\prime 2}\right\rangle\langle u\rangle_{x}-\left\langle u^{\prime} v^{\prime}\right\rangle\left(\langle u\rangle_{y}+\langle v\rangle_{x}\right) \\
& -\left\langle v^{\prime 2}\right\rangle\langle\boldsymbol{v}\rangle_{y}-\left\langle\mathbf{v}^{h^{\prime}} w^{\prime}\right\rangle\left\langle\mathbf{v}^{h}\right\rangle_{z},
\end{aligned}
$$

which are respectively the product of horizontal mean shear and horizontal Reynolds stress (HRS) of the Eulerian field, and the product of vertical mean shear and vertical Reynolds stress (VRS) of the Eulerian field. The latter is also referred to as shear production, and their WEC counterparts as Stokes shear production. This term represents the energy conversion from the mean, noted with $\langle\cdot\rangle$ and defined as the horizontal average at $z$ levels, to the perturbation, noted with a prime $(')$. The buoyancy production, also commonly called $P_{e} K_{e}$, is

$$
P_{e} K_{e}=\left\langle w^{\prime} b^{\prime}\right\rangle
$$

and the mean potential to mean kinetic energy conversion is

$$
P_{m} K_{m}=\langle w\rangle\langle b\rangle .
$$

With WEC additional energy conversion terms are 

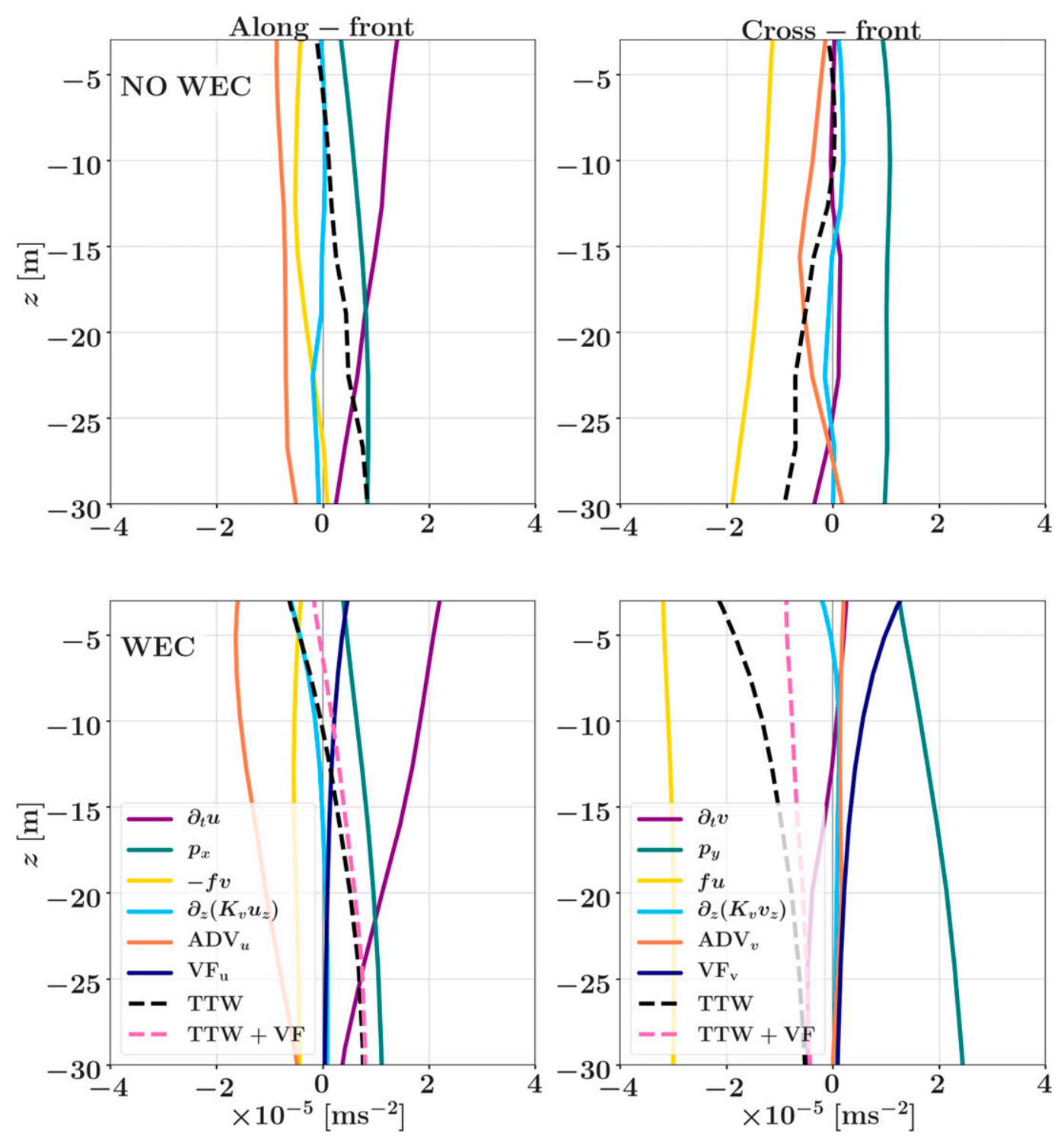

FIG. 10. Vertical profiles of alongfront-averaged left-side terms of the momentum balance (7) in the along- and cross-front directions of the filament identified in Fig. 8 during event 1 . An additional horizontal averaging is done within the vicinity of the frontal location (averaging-window width $\simeq 1.1 \mathrm{~km}$ ): (top) the solution without WEC and (bottom) the solution with WEC. Residuals of the TTW balance are black dashed lines, residuals of a combined TTW plus the vortex force are pink dashed lines. The terms displayed, in the along and cross directions, are the pressure gradient $p_{x}, p_{y}$, the Coriolis force $-f v, f u$, the vertical mixing term $\partial_{z}\left(K_{v} u_{z}\right), \partial_{z}\left(K_{v} v_{z}\right)$, the advection term $\mathrm{ADV}_{u}, \mathrm{ADV}_{v}$, and the vortex force $\mathrm{VF}_{u}, \mathrm{VF}_{v}$.

$$
\begin{aligned}
\mathrm{HRS}^{\mathrm{St}}+\mathrm{VRS}^{\mathrm{St}}= & -\left\langle u^{\prime 2}\right\rangle\left\langle u^{\mathrm{St}}\right\rangle_{x}-\left\langle u^{\prime} v^{\prime}\right\rangle\left(\left\langle u^{\mathrm{St}}\right\rangle_{y}\right. \\
& \left.+\left\langle v^{\mathrm{St}}\right\rangle_{x}\right)-\left\langle v^{\prime 2}\right\rangle\left\langle v^{\mathrm{St}}\right\rangle_{y}-\left\langle\mathbf{v}^{h^{\prime}} w^{\prime}\right\rangle\left\langle\mathbf{v}^{h \mathrm{St}}\right\rangle_{z} .
\end{aligned}
$$

For both Eulerian and Stokes fields, there is a natural separation of scales such as all the above expressions have been simplified considering that $w \ll u, v$ and $\partial_{z} \gg \partial_{x}, \partial_{y}$. In LES for Langmuir Turbulence, it is commonly assumed that Stokes drift only varies with depth, and therefore the HRS ${ }^{\mathrm{St}}$ vanishes and only the shear production terms remain, e.g., Suzuki and
Fox-Kemper (2016). This means that usually Stokes drift are considered both horizontally smooth and their departure to a spatial and/or temporal mean is very small $\left(\mathbf{v}^{h \mathrm{St} t} \ll 1\right)$. Here, we compute HRS $^{\text {St }}$ and recover this behavior (see the light blue curve in Fig. 14), i.e., HRS $^{\text {St }}$ is always small in our simulations and in agreement with LT dynamics.

In Fig. 14 we show the time series of these energy conversion terms with a decomposition using horizontal averaging within the dashed box in Fig. 1 at $z$ levels as the mean flow and fluctuations around it. We vertically integrate over the first $30 \mathrm{~m}$ and show the results during the two high-wave events of 


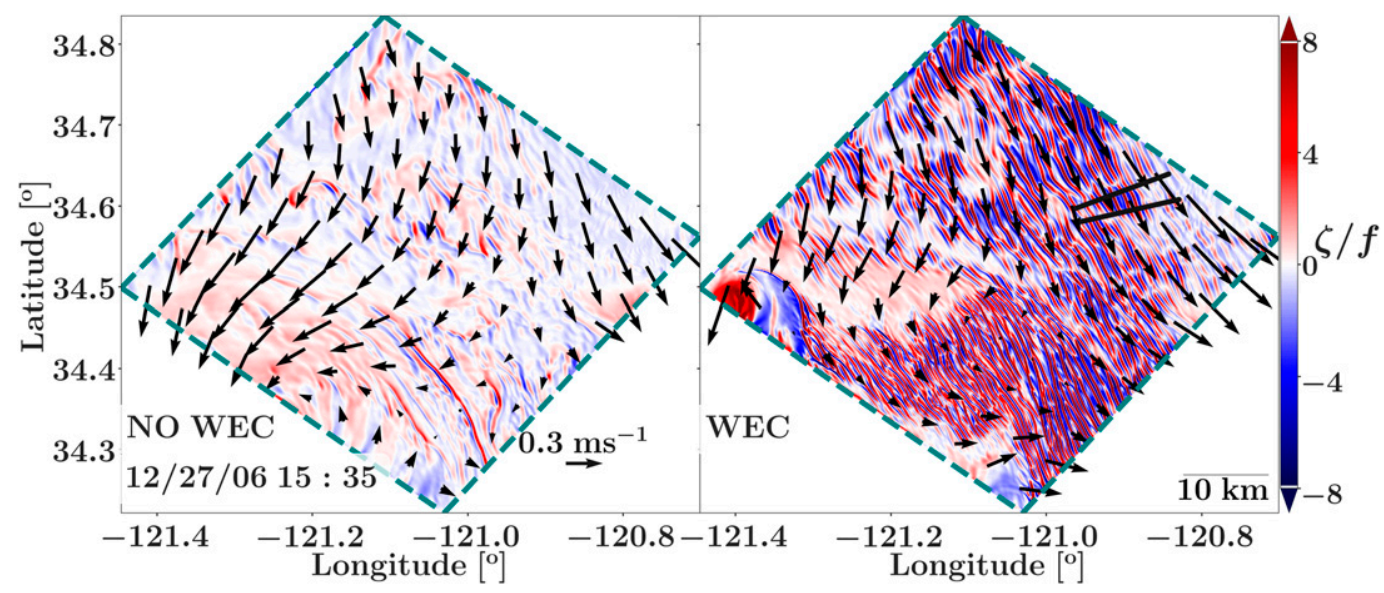

FIG. 11. Snapshots of normalized surface vorticity from the L3 solutions (right) with and (left) without WEC. The time is 1535 local time $27 \mathrm{Dec} 2006$ (event 2). Surface Lagrangian surface currents are superimposed as arrows. The black lines demarcate the portion of the roll-cell structures we use when averaging.

December 2006. During event 1 , the buoyancy production and vertical shear $\left(P_{e} K_{e}\right.$ and VRS) terms are negligible, and event 1 is dominated by a combination of the conversions HRS (Eulerian) and $P_{m} K_{m}$. This is consistent with submesoscale frontogenesis (led by positive $P_{m} K_{m}$ ) and frontal instability (led by HRS) dynamics as analyzed by Gula et al. (2014). Submesoscale features are primarily controlled by these two sources: HRS from the Eulerian field and $P_{m} K_{m}$. When averaging over the domain (sampled areas in Fig. 1), we find them opposed in sign. This illustrates that frontogenesis (peak of $P_{m} K_{m}$ ) and frontal arrest and decay (when HRS grows while $P_{m} K_{m}$ decays) operate at different times (Sullivan and McWilliams 2018), although the multiple cycles of opposing sign are not entirely familiar or explained. The evolution of $P_{m} K_{m}$ and HRS in the time series of Fig. 14 likely reflects the averaging over a population of fronts and filaments each at distinct stages in their life cycle. In spite of WEC influences being significant in other balances (section 4), they are not important as direct conversion terms in the EKE balance.

During event 2 the energy conversions are quite different. The Eulerian and Stokes shear production VRS terms are strong sources of EKE for the roll cells, and the Eulerian HRS term is a sink. The large Eulerian shear production VRS term comes from the large vertical shear of the background flow during event 2. Similarly, the large Stokes VRS comes from the vertical shear of the Stokes drift. Together, these vertical shears are responsible for the dominance of $-\left\langle u^{\prime} w^{\prime}\right\rangle\left\langle u+u^{\mathrm{St}}\right\rangle_{z}>0$ at that time. The amplitude of the Eulerian HRS is primarily controlled by the term $-\left\langle u^{\prime 2}\right\rangle\langle u\rangle_{x}$ where both $\left\langle u^{\prime 2}\right\rangle$ and $\langle u\rangle_{x}$ are positive and enhanced during event 2. But, the Eulerian HRS is related to the background larger-scale flow and does not seem strongly associated with
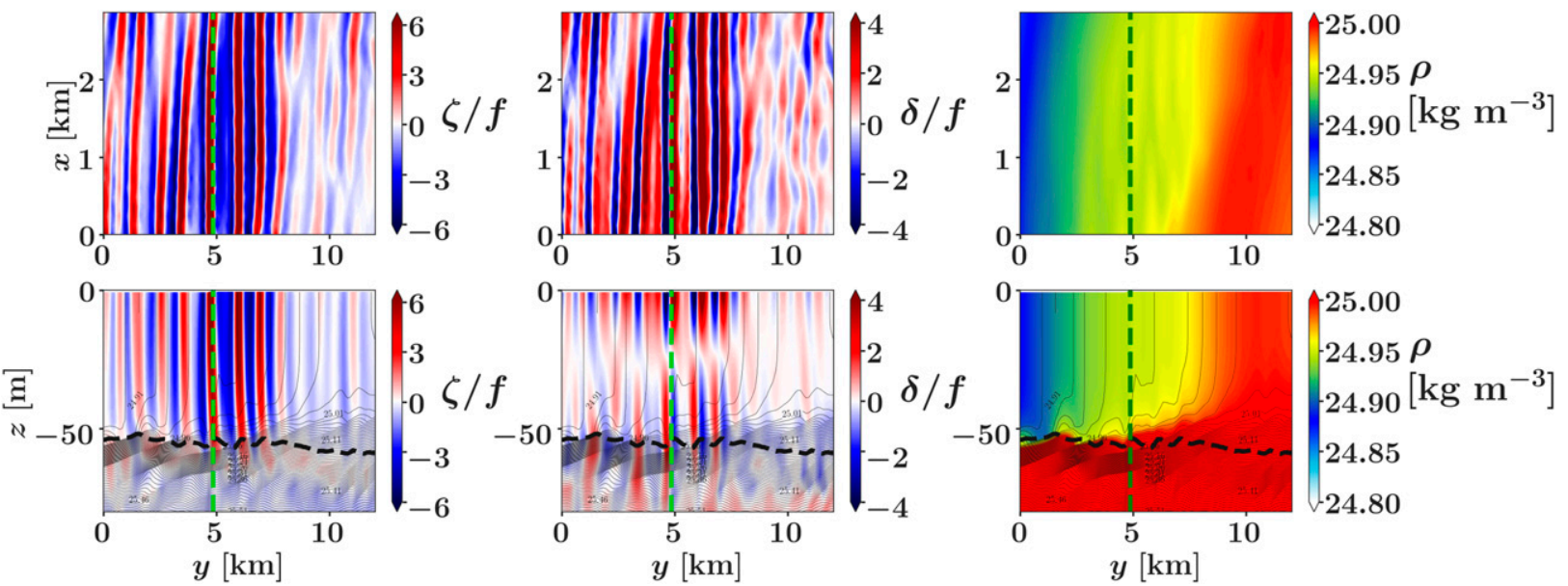

FIG. 12. (top) Surface maps and (bottom) cross-front vertical sections of averaged normalized (left) vorticity, (center) divergence, and (right) density across the roll-cell structures, at 1535 local time 27 Dec 2006 (during event 2). Isopycnals and mixed layer depth (dashed) are plotted in black lines. The contour interval of the isopycnals is $0.01 \mathrm{~kg} \mathrm{~m}^{-3}$. The dashed green line is at the same location in this figure and in Fig. 13. 

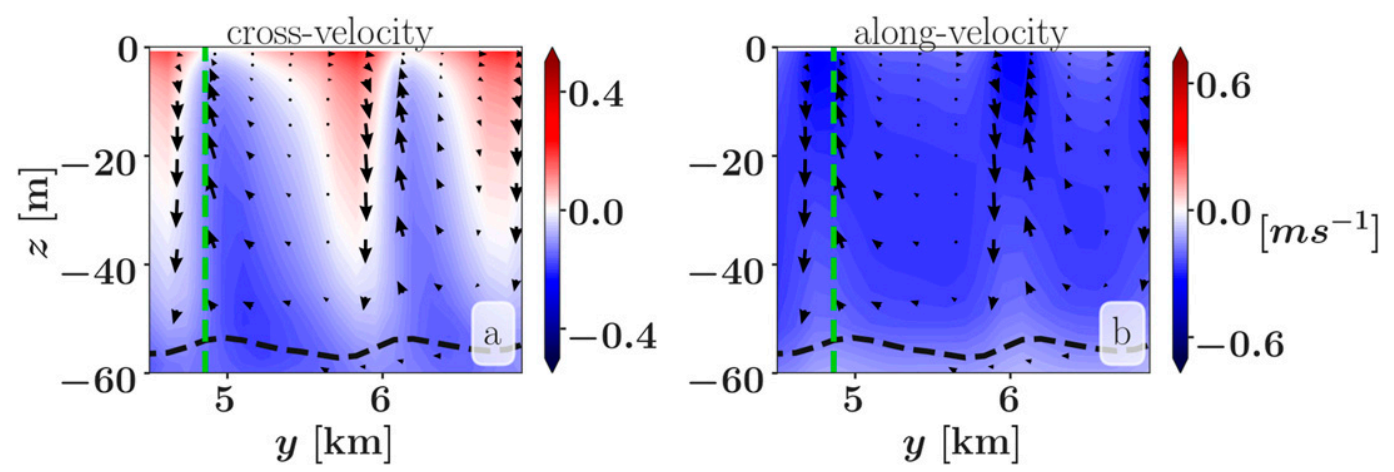

FIG. 13. (a) Cross-velocity and (b) along-velocity (in colors), cross and vertical velocities (vectors), across two roll-cell structures, at 1535 local time 27 Dec 2006 (during event 2). Vertical velocities have been amplified by $10^{2}$. The mixed layer depth is plotted in dashed black lines. The dashed green line is at the same location in this figure and in Fig. 12.

the roll cells in this area average analysis. This suggests that the roll cells arise from a vertical shear instability of the ambient flow, while there is some return of energy by the horizontal Reynolds stress, which may not be a generic behavior for WEC-induced roll cells. This is quite different from the familiar submesoscale frontogenesis regime, which seems to be locally inhibited here when roll cells are active. However, this is consistent with the systematic larger submesoscale weakening we observe during this time (section 4), where we conclude that differential advection from the Eulerian field $B_{\text {hor }}$ is responsible for a sink of the squared buoyancy gradient. The roll cells' emergence is characterized by a growing positive $\mathrm{VRS}^{\mathrm{St}}$ term, of leading order in the energy balance, in agreement with LT dynamics.

In summary, there are similarities between the submesoscale roll cells simulated here and Langmuir circulations in LT, although the former has much larger length scales $L$. While our resolution limitations preclude making a definitive distinction at this time, we conclude that there is likely a previously numerically unresolved roll-cell mode of submesoscale currents beyond the familiar wind-wave LT regime.

\section{Summary and discussion}

The present study focuses on the interactions between surface gravity waves and mesoscale and submesoscale currents in realistic simulations using ROMS with WRF- and WW3derived forcings. These simulations implement a recent development of an improved approximation of the Stokes drift (Romero et al. 2021) that produces Stokes drift amplitudes in good agreement with WW3 full spectrum integration and not restricted to deep-water waves. We perform a systematic comparison of WEC with NO WEC solutions (without wave forcing) and of a horizontal grid resolution sensitivity (grids L2 and L3 here).

Statistical differences between solutions with and without WEC arise when the averaged wave amplitude is larger than about $1.5 \mathrm{~m}\left(H_{s}>4.2 \mathrm{~m}\right)$. In such wave conditions WEC statistically intensifies near-surface velocity and density gradients in the submesoscale regime. The submesoscale dynamics maintain cyclonic and convergence preferences but with larger magnitudes. Dynamical balance analyses show that large amplitude remote swells can trigger a source of vertical shear, through the vertical shear of the Stokes drift, that leads to the increase of surface vertical enstrophy. Large wind-forced waves enhance vertical enstrophy through the same mechanism, but additionally feed a sink of surface buoyancy gradients through the onset of very smallscale structures associated with large horizontal gradients of vertical velocities. The source of vertical shear associated with the vertical shear of the Stokes drift is directly derived from the vortex force. We confirm in an instantaneous
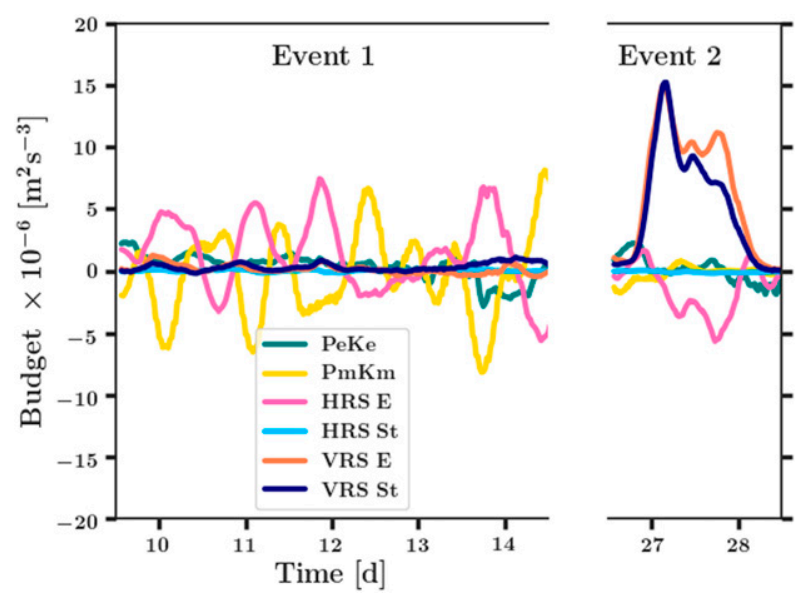

FIG. 14. Time series (days since 0000 local time 1 Dec 2006, ) of EKE budget conversion terms from (10), (11), (12), and (13). Vertical (VRS) and horizontal (HRS) Reynolds stress terms have two counterparts: St is the conversion due to the Stokes drift, and $E$ is the conversion due to the Eulerian field. The buoyancy production is $P_{e} K_{e}$ (eddy potential to eddy kinetic energy conversion), and $P_{m} K_{m}$ is the mean potential to mean kinetic energy conversion term. Fields are horizontally averaged in the sampled areas shown in Fig. 1 and vertically integrated over the first $30 \mathrm{~m}$ for the L3 WEC solution during the two large-wave-amplitude events of December 2006. 
momentum balance analysis that the vortex force plays an important role in both the familiar submesoscale fronts and filaments and the smaller submesoscale roll cells.

These roll cells of positive and negative vorticity and divergence are a previously unresolved simulated submesoscale feature that is most evident in the large-amplitude wave regime associated with strong wind. They are associated with large vertical Lagrangian shear production in the KE budget, reminiscent of Langmuir cells, but here with the hydrostatic approximation. However, the horizontal and vertical scales of these structures and their alignment with the Lagrangian surface current $\left(\mathbf{v}^{h}+\mathbf{v}^{h \mathrm{St}}\right)$ complicates their present interpretation. We leave further examination of this phenomenon to future, higher-resolution simulations that can also include nonhydrostatic dynamics in ROMS (Roullet et al. 2017).

This study focuses exclusively on two large-amplitude events during December 2006 and thus lacks seasonal variability. This choice is deliberate, as the Northern Hemisphere winter period captures more energetic weather (wind and wave) and submesoscale events and therefore possibly displays more dramatic WEC consequences. We expect periods with calmer conditions (summer, for instance) to display similar, yet smaller WEC effects.

The small number of large-wave events in this study (two) makes it difficult as yet to establish a universal criterion quantifying the importance of WEC on ocean dynamics. These events were initially identified by a large wave amplitude ( $A_{\text {wave }} \gtrsim 1.5 \mathrm{~m} ; H_{s} \gtrsim 4.2 \mathrm{~m}$ ) and a large Stokes drift, $\gtrsim 0.1 \mathrm{~m} \mathrm{~s}^{-1}$. Seeking nondimensional measures, a comparison with the local wind forcing using the turbulent Langmuir number $\left(\mathrm{La}_{t}\right)$ in (9) shows that it becomes very small during both events $(\leq 0.3)$. However, other comparisons of Stokes drift with Eulerian current measures were not as successful in distinguishing these two events from other periods, even though their anomalous WEC response is clear from the outcome (Fig. 2, lower panels).

Even though an analysis of the larger domain in L2 shows a clear WEC signal on the shelf and near the shore, here we have deliberately left aside the surface wave interactions in the surfzone and inner-shelf by investigating solely open-ocean dynamics in deep water. We hope that particular cases of possible shallow water, coastal interactions and large wave-breaking associated with momentum inputs to currents will be the focus of future investigations with finer grid resolution.

Acknowledgments. We thank Jeroen Molemaker, Kaushik Srinivasan, Peng Wang, and Yusuke Uchiyama for many helpful discussions, as well as Lionel Renault, Fayçal Kessouri, and Pierre Damien for providing the parent WRF atmospheric forcing and L1 solution used in our nesting procedure and Çiğdem Akan for her exploratory simulations in this region. This work used the XSEDE supercompter (Towns et al. 2014) Comet through the allocation TG-OCE027000N. This research is supported by the National Science Foundation (NSF, Grant OCE-1355970) and the U.S. Office of Naval Research (ONR, Grants N00014-14-1-0626 and N00014-15-1-2645). LR was supported by grants from ONR N00014-16-1-2936 and NSF OCE-1924686.

\section{REFERENCES}

Barkan, R., M. J. Molemaker, K. Srinivasan, J. C. McWilliams, and E. A. D'Asaro, 2019: The role of horizontal divergence in submesoscale frontogenesis. J. Phys. Oceanogr., 49, 1593-1618, https://doi.org/10.1175/JPO-D-18-0162.1.

Belcher, S. E., and Coauthors, 2012: A global perspective on Langmuir turbulence in the ocean surface boundary layer. Geophys. Res. Lett., 39, L18605, https://doi.org/10.1029/ 2012 GL052932.

Bracco, A., J. Choi, K. Joshi, H. Luo, and J. C. McWilliams, 2016: Submesoscale currents in the northern Gulf of Mexico: Deep phenomena and dispersion over the continental slope. Ocean Modell., 101, 43-58, https://doi.org/10.1016/j.ocemod.2016.03.002.

Breivik, Ø., P. A. Janssen, and J.-R. Bidlot, 2014: Approximate Stokes drift profiles in deep water. J. Phys. Oceanogr., 44, 2433-2445, https://doi.org/10.1175/JPO-D-14-0020.1.

Buijsman, M., Y. Uchiyama, J. McWilliams, and C. Hill-Lindsay, 2012: Modeling semidiurnal internal tide variability in the Southern California Bight. J. Phys. Oceanogr., 42, 62-77, https://doi.org/10.1175/2011JPO4597.1.

Callies, J., R. Ferrari, J. M. Klymak, and J. Gula, 2015: Seasonality in submesoscale turbulence. Nat. Commun., 6, 6862, https:// doi.org/10.1038/ncomms7862.

Capet, X., J. C. McWilliams, M. J. Molemaker, and A. F. Shchepetkin, 2008a: Mesoscale to submesoscale transition in the California Current System. Part I: Flow structure, eddy flux, and observational tests. J. Phys. Oceanogr., 38, 29-43, https://doi.org/10.1175/2007JPO3671.1.

,,-- , and A. Shchepetkin, 2008b: Mesoscale to submesoscale transition in the California Current System. Part II: Frontal processes. J. Phys. Oceanogr., 38, 44-64, https:// doi.org/10.1175/2007JPO3672.1.

,,--- , and A. F. Shchepetkin, 2008c: Mesoscale to submesoscale transition in the California Current System. Part III: Energy balance and flux. J. Phys. Oceanogr., 38, 22562269, https://doi.org/10.1175/2008JPO3810.1.

Charney, J. G., 1971: Geostrophic turbulence. J. Atmos. Sci., 28, 1087-1095, https://doi.org/10.1175/1520-0469(1971)028<1087: $\mathrm{GT}>2.0 . \mathrm{CO} ; 2$.

Craik, A. D., and S. Leibovich, 1976: A rational model for Langmuir circulations. J. Fluid Mech., 73, 401-426, https:// doi.org/10.1017/S0022112076001420.

Dauhajre, D. P., and J. C. McWilliams, 2018: Diurnal evolution of submesoscale front and filament circulations. J. Phys. Oceanogr., 48, 2343-2361, https://doi.org/10.1175/JPO-D18-0143.1.

_ _ — , and L. Renault, 2019: Nearshore Lagrangian connectivity: Submesoscale influence and resolution sensitivity. J. Geophys. Res. Oceans, 124, 5180-5204, https://doi.org/ 10.1029/2019JC014943.

Feddersen, F., and J. Trowbridge, 2005: The effect of wave breaking on surf-zone turbulence and alongshore currents: A modeling study. J. Phys. Oceanogr., 35, 2187-2203, https:// doi.org/10.1175/JPO2800.1.

Gnanadesikan, A., and R. A. Weller, 1995: Structure and instability of the Ekman spiral in the presence of surface gravity waves. J. Phys. Oceanogr., 25, 3148-3171, https://doi.org/10.1175/ 1520-0485(1995)025<3148:SAIOTE $>2.0$. CO 2 .

Goodman, L., 2012: Scalable lateral mixing and coherent turbulence DRI: Use of an AUV to quantify submesoscale mixing processes. University of Massachusetts Dartmouth School for Marine Science and Technology Tech. Rep. ADA531919, 8 pp., https://apps.dtic.mil/sti/citations/ADA531919. 
Gula, J., M. J. Molemaker, and J. C. McWilliams, 2014: Submesoscale cold filaments in the Gulf Stream. J. Phys. Oceanogr., 44, 26172643, https://doi.org/10.1175/JPO-D-14-0029.1.

Hamlington, P. E., L. P. Van Roekel, B. Fox-Kemper, K. Julien, and G. P. Chini, 2014: Langmuir-submesoscale interactions: Descriptive analysis of multiscale frontal spindown simulations. J. Phys. Oceanogr., 44, 2249-2272, https://doi.org/ 10.1175/JPO-D-13-0139.1.

Hanley, K. E., S. E. Belcher, and P. P. Sullivan, 2010: A global climatology of wind-wave interaction. J. Phys. Oceanogr., 40, 1263-1282, https://doi.org/10.1175/2010JPO4377.1.

Harcourt, R. R., and E. A. D'Asaro, 2008: Large-eddy simulation of Langmuir turbulence in pure wind seas. J. Phys. Oceanogr., 38, 1542-1562, https://doi.org/10.1175/2007JPO3842.1.

Kraichnan, R. H., 1967: Inertial ranges in two-dimensional turbulence. Phys. Fluids, 10, 1417-1423, https://doi.org/10.1063/ 1.1762301 .

Kumar, N., G. Voulgaris, J. C. Warner, and M. Olabarrieta, 2012: Implementation of the vortex force formalism in the Coupled Ocean-Atmosphere-Wave-Sediment Transport (COAWST) modeling system for inner shelf and surf zone applications. Ocean Modell., 47, 65-95, https://doi.org/10.1016/j.ocemod.2012.01.003.

Langmuir, I., 1938: Surface motion of water induced by wind. Science, 87, 119-123, https://doi.org/10.1126/science.87.2250.119.

Large, W. B., 2006: Surface fluxes for practitioners of global ocean data assimilation. Ocean Weather Forecasting, Springer, 229-270.

Large, W. G., J. C. McWilliams, and S. C. Doney, 1994: Oceanic vertical mixing: A review and a model with a nonlocal boundary layer parameterization. Rev. Geophys., 32, 363-403, https://doi.org/10.1029/94RG01872.

Lemarié, F., J. Kurian, A. F. Shchepetkin, M. Jeroen Molemaker, F. Colas, and J. C. McWilliams, 2012: Are there inescapable issues prohibiting the use of terrain-following coordinates in climate models? Ocean Modell., 42, 57-79, https://doi.org/ 10.1016/j.ocemod.2011.11.007.

Lévy, M., R. Ferrari, P. J. S. Franks, A. P. Martin, and P. Rivière, 2012: Bringing physics to life at the submesoscale. Geophys. Res. Lett., 39, L14602, https://doi.org/10.1029/2012GL052756.

Li, Q., and B. Fox-Kemper, 2017: Assessing the effects of Langmuir turbulence on the entrainment buoyancy flux in the ocean surface boundary layer. J. Phys. Oceanogr., 47, 2863-2886, https://doi.org/10.1175/JPO-D-17-0085.1.

— , and Coauthors, 2019: Comparing ocean surface boundary vertical mixing schemes including Langmuir Turbulence. J. Adv. Model. Earth Syst., 11, 3545-3592, https://doi.org/ 10.1029/2019MS001810.

Longuet-Higgins, M. S., and R. W. Stewart, 1964: Radiation stresses in water waves: A physical discussion, with applications. Deep-Sea Res., 11, 529-562, https://doi.org/10.1016/ 0011-7471(64)90001-4.

MacMahan, J. H., E. B. Thornton, and A. J. Reniers, 2006: Rip current review. Coast. Eng., 53, 191-208, https://doi.org/ 10.1016/j.coastaleng.2005.10.009.

Mahadevan, A., 2016: The impact of submesoscale physics on primary productivity of plankton. Annu. Rev. Mar. Sci., 8, 161184, https://doi.org/10.1146/annurev-marine-010814-015912.

— mesoscale vertical motion at ocean fronts. Ocean Modell., 14, 241-256, https://doi.org/10.1016/j.ocemod.2006.05.006.

Marchesiello, P., R. Benshila, R. Almar, Y. Uchiyama, J. C. McWilliams, and A. Shchepetkin, 2015: On tridimensional rip current modeling. Ocean Modell., 96, 36-48, https://doi.org/ 10.1016/j.ocemod.2015.07.003.
Marmorino, G., G. Smith, and G. Lindemann, 2005: Infrared imagery of large-aspect-ratio Langmuir circulation. Cont. Shelf Res., 25, 1-6, https://doi.org/10.1016/j.csr.2004.08.002.

Mason, E., J. Molemaker, A. F. Shchepetkin, F. Colas, J. C. McWilliams, and P. Sangrà, 2010: Procedures for offline grid nesting in regional ocean models. Ocean Modell., 35, 1-15, https://doi.org/10.1016/j.ocemod.2010.05.007.

McWilliams, J. C., 2016: Submesoscale currents in the ocean. Proc. Math. Phys. Eng. Sci., 472, 20160117, https://doi.org/10.1098/ rspa.2016.0117.

- 2018: Surface wave effects on submesoscale fronts and filaments. J. Fluid Mech., 843, 479-517, https://doi.org/10.1017/ jfm.2018.158.

_ _ P. P. Sullivan, and C.-H. Moeng, 1997: Langmuir turbulence in the ocean. J. Fluid Mech., 334, 1-30, https://doi.org/10.1017/ S0022112096004375.

_ J. M. Restrepo, and E. M. Lane, 2004: An asymptotic theory for the interaction of waves and currents in coastal waters. J. Fluid Mech., 511, 135-178, https://doi.org/10.1017/S0022112004009358.

- E. Huckle, J. Liang, and P. P. Sullivan, 2014: Langmuir turbulence in swell. J. Phys. Oceanogr., 44, 870-890, https:// doi.org/10.1175/JPO-D-13-0122.1.

Michalakes, J., J. Dudhia, D. Gill, J. Klemp, and W. Skamarock, 1998: Design of a next-generation regional weather research and forecast model. Towards Teracomputing, World Scientific, 117-124.

Nagai, T., A. Tandon, and D. L. Rudnick, 2006: Two-dimensional ageostrophic secondary circulation at ocean fronts due to vertical mixing and large-scale deformation. J. Geophys. Res., 111, C09038, https://doi.org/10.1029/2005JC002964.

Olabarrieta, M., J. C. Warner, and N. Kumar, 2011: Wave-current interaction in Willapa Bay. J. Geophys. Res., 116, C12014, https://doi.org/10.1029/2011JC007387.

Renault, L., M. J. Molemaker, J. C. McWilliams, A. F. Shchepetkin, F. Lemarié, D. Chelton, S. Illig, and A. Hall, 2016: Modulation of wind work by oceanic current interaction with the atmosphere. J. Phys. Oceanogr., 46, 1685-1704, https://doi.org/ 10.1175/JPO-D-15-0232.1.

—, J. C. McWilliams, F. Kessouri, A. Jousse, H. Frenzel, R. Chen, and C. Deutsch, 2021: Evaluation of high-resolution atmospheric and oceanic simulations of the California Current System. Prog. Oceanogr., 195, 102564, https://doi.org/10.1016/ j.pocean.2021.102564.

Romero, L., Y. Uchiyama, J. C. Ohlmann, J. C. McWilliams, and D. A. Siegel, 2013: Simulations of nearshore particle-pair dispersion in Southern California. J. Phys. Oceanogr., 43, 1862-1879, https://doi.org/10.1175/JPO-D-13-011.1.

—, D. Hypolite, and J. C. McWilliams, 2020: Submesoscale current effects on surface waves. Ocean Modell., 153, 101662, https://doi.org/10.1016/j.ocemod.2020.101662.

,-- , and,- 2021 : Representing wave effects on currents. Ocean Modell., 167, 101873, https://doi.org/10.1016/ j.ocemod.2021.101873.

Roullet, G., J. M. Molemaker, N. Ducousso, and T. Dubos, 2017: Compact symmetric Poisson equation discretization for non-hydrostatic sigma coordinates ocean model. Ocean Modell., 118, 107-117, https://doi.org/10.1016/ j.ocemod.2017.09.001.

Shchepetkin, A. F., and J. C. McWilliams, 2005: The Regional Oceanic Modeling System (ROMS): A split-explicit, freesurface, topography-following-coordinate oceanic model. Ocean Modell., 9, 347-404, https://doi.org/10.1016/ j.ocemod.2004.08.002. 
Srinivasan, K., J. C. McWilliams, and A. Jagannathan, 2021: High vertical shear and dissipation in equatorial topographic wakes. J. Phys. Oceanogr, 51, 1985-2001, https://doi.org/10.1175/JPOD-20-0119.1.

Sullivan, P. P., and J. C. McWilliams, 2010: Dynamics of winds and currents coupled to surface waves. Annu. Rev. Fluid Mech., 42, 19-42, https://doi.org/10.1146/annurev-fluid-121108-145541.

— , and _ 2018: Frontogenesis and frontal arrest for a dense filament in the oceanic surface boundary layer. J. Fluid Mech., 837, 341-380, https://doi.org/10.1017/jfm.2017.833. , and - 2019: Langmuir turbulence and filament frontogenesis in the oceanic surface boundary layer. J. Fluid Mech., 879, 512-553, https://doi.org/10.1017/jfm.2019.655.

, L. Romero, J. C. McWilliams, and W. K. Melville, 2012: Transient evolution of Langmuir turbulence in ocean boundary layers driven by hurricane winds and waves. J. Phys. Oceanogr., 42, 1959-1980, https://doi.org/10.1175/JPO-D-12025.1 .

Sun, D., and Coauthors, 2020: Diurnal cycling of submesoscale dynamics: Lagrangian implications in drifter observations and model simulations of the northern Gulf of Mexico. J. Phys. Oceanogr., 50, 1605-1623, https://doi.org/10.1175/JPO-D-190241.1.

Sundermeyer, M. A., E. Skyllingstad, J. R. Ledwell, B. Concannon, E. A. Terray, D. Birch, S. D. Pierce, and B. Cervantes, 2014: Observations and numerical simulations of large-eddy circulation in the ocean surface mixed layer. Geophys. Res. Lett., 41, 7584-7590, https://doi.org/10.1002/2014GL061637.

Suzuki, N., and B. Fox-Kemper, 2016: Understanding Stokes forces in the wave-averaged equations. J. Geophys. Res. Oceans, 121, 3579-3596, https://doi.org/10.1002/2015JC011566.
Tamura, H., Y. Miyazawa, and L.-Y. Oey, 2012: The Stokes drift and wave induced-mass flux in the North Pacific. J. Geophys. Res., 117, C08021, https://doi.org/10.1029/2012JC008113.

Thorpe, S., 2004: Langmuir circulation. Annu. Rev. Fluid Mech., 36, 55-79, https://doi.org/10.1146/annurev.fluid.36.052203.071431.

Tolman, H. L., 2009: User manual and system documentation of WAVEWATCH III TM version 3.14. Tech. Note MMAB Contribution 276, 220 pp.

Towns, J., and Coauthors, 2014: XSEDE: Accelerating scientific discovery. Comput. Sci. Eng., 16, 62-74, https://doi.org/10.1109/ MCSE.2014.80.

Uchiyama, Y., J. C. McWilliams, and A. F. Shchepetkin, 2010: Wave-current interaction in an oceanic circulation model with a vortex-force formalism: Application to the surf zone. Ocean Modell., 34, 16-35, https://doi.org/ 10.1016/j.ocemod.2010.04.002.

— — - and C. Akan, 2017: Three-dimensional transient rip currents: Bathymetric excitation of low-frequency intrinsic variability. J. Geophys. Res. Oceans, 122, 5826-5849, https:// doi.org/10.1002/2017JC013005.

Van Roekel, L. P., B. Fox-Kemper, P. P. Sullivan, P. E. Hamlington, and S. R. Haney, 2012: The form and orientation of Langmuir cells for misaligned winds and waves. J. Geophys. Res., 117, C05001, https://doi.org/10.1029/2011JC007516.

Wang, T., R. Barkan, J. C. McWilliams, and M. J. Molemaker, 2021: Structure of Submesoscale Fronts of the Mississippi River Plume. J. Phys. Oceanogr., 51, 1113-1131, https:// doi.org/10.1175/JPO-D-20-0191.1.

Zhong, Y., and A. Bracco, 2013: Submesoscale impacts on horizontal and vertical transport in the Gulf of Mexico. J. Geophys. Res. Oceans, 118, 5651-5668, https://doi.org/10.1002/jgrc.20402. 